\title{
Multi-Target Counting and Localization in Distributed MIMO Radar Based on Energy Modeling and Compressive Sensing
}

This paper was downloaded from TechRxiv (https://www.techrxiv.org).

\section{LICENSE}

CC BY 4.0

SUBMISSION DATE / POSTED DATE

03-03-2022 / 08-03-2022

\section{CITATION}

Zhu, Lingxiao; Wen, Gongjian; Liang, Yuanyuan; Luo, Dengsanlang; Jian, Haojun (2022): Multi-Target Counting and Localization in Distributed MIMO Radar Based on Energy Modeling and Compressive Sensing. TechRxiv. Preprint. https://doi.org/10.36227/techrxiv.19295369.v1

$\mathrm{DOI}$ 


\title{
Multi-Target Counting and Localization in Distributed MIMO Radar Based on Energy Modeling and Compressive Sensing
}

\author{
Lingxiao Zhu, Gongjian Wen, Member, IEEE, Yuanyuan Liang, Dengsanlang Luo, and Haojun Jian
}

\begin{abstract}
Target localization is one of the most important research topics in the field of radar signal processing. In this paper, the problem of multi-target counting and localization in the distributed multiple-input multiple-output (MIMO) radar is investigated. We first analyze the theoretical bound of the multitarget localization accuracy in the discrete time signal model. It is determined by the Cramér-Rao lower bound (CRLB) at low signal-to-noise ratio (SNR) and the sampling lower bound (SLB) when the SNR is high. Furthermore, an innovative multitarget counting and localization scheme is developed, which is based on the energy modeling of the multiple transmitter-receiver paths and the compressive sensing theory. To solve the sparse vector recovery issue, we design a lightweight iterative greedy pursuit algorithm including the similarity evaluation strategy. The proposal utilizes the samples of the raw signals and belongs to the category of the direct localization. Nevertheless, it has significantly higher computational efficiency and lower communication burden than the conventional direct localization methods, while avoids the complex data association that encountered by the indirect localization methods. Finally, the simulation results validate the effectiveness and robustness of the proposed method.
\end{abstract}

Index Terms-Distributed multiple-input multiple-output (MIMO) radar, multi-target counting, multi-target localization, Cramér-Rao lower bound (CRLB), sampling lower bound (SLB), energy modeling, compressive sensing.

\section{INTRODUCTION}

$\mathbf{M}$ ULTIPLE-INPUT multiple-output (MIMO) radar has received considerable research interest in recent years [1]-[10]. In general, there are two main categories of the MIMO radar. The first is the colocated MIMO radar [2], which consists of transmit and receive arrays with colocated elements, and can exploit multiple independent signals to acquire the waveform diversity. The second is the MIMO radar with the widely separated antennas, also known as the distributed MIMO radar [3]. As its name implies, this type of radar is composed of multiple transmitters and receivers that are spatially distributed. It takes full advantage of the spatial and geometric diversity, so that the target detection and localization performance can be improved. A lot of works have

This work is supported by the National Natural Science Foundation of China (NSFC) under Grant No. 61902420. (Corresponding author: Lingxiao Zhu.)

L. Zhu, G. Wen Y. Liang, and D. Luo are with the National Key Laboratory of Science and Technology on ATR, College of Electronic Science, National University of Defense Technology, Changsha 410073, China (email: LingxiaoZ@yeah.net; wengongjian@sina.com; lyy12151119@163.com; ldengsanlang@sina.com).

H. Jian is with School of Geodesy and Geomatics, Wuhan University, Wuhan 430079, China (e-mail: 2018302141225@whu.edu.cn). studied the problems with regard to the distributed MIMO radar so far, including target detection [1], [11]-[15], target localization [5], [7], [16]-[18], optimal antenna placement [19], [20], resource allocation [21], etc. In this paper, we discuss the topic of simultaneously determining the number and the positions of multiple targets based on the distributed MIMO radar, that is, addressing the multi-target counting and localization problem.

From the point of view of the signal processing difference, the target localization methods based on the distributed MIMO radar can be divided into the indirect manner and the direct manner. The indirect approaches are usually executed in two steps. First, the specific intermediate measurement of each transmitter-receiver path is estimated, such as time delay, angle of arrival, and Doppler shift. Second, the target parameters are estimated by fusing the intermediate measurements of multiple paths. Basically, the solutions for the indirect target localization can also be divided into two classes. The first class solves the problem in the closed form. For example, a localization method by using the least squares (LS) estimation is developed in [16]. In [17], the method based on the idea of two-stage weighted least squares (TSWLS) is designed, which utilizes the virtual grouping and weighted fusion strategy. Moreover, some other closed form solutions are developed in [22]-[24]. The second class addresses the issue via some optimization tools. In [25], the target localization problem is solved by the Lagrange programming neural network (LPNN), which is an effective tool for the nonlinear constrained optimization problems. In [26], the half-quadratic and the semidefinite relaxation (SDR) techniques are used to deal with the localization problem. This class of approaches are also investigated in [27], [28]. Overall, the indirect localization methods can produce satisfactory precision when the signal-to-noise ratio (SNR) of each transmitter-receiver path is sufficiently high. Besides, most of the indirect methods are computational fast, especially those solutions in the closed form. However, there also exist intrinsic drawbacks for these methods. For instance, the localization performance may suffer serious degradation when the SNR is low, and the precise data association is also intractable if there are multiple targets.

The direct localization methods estimate the target parameters based on the received signals directly. The typical approaches first form the objective function according to the maximum-likelihood (ML) estimation determined by the signal model, and then invoke the multiple dimensional search and some standard optimization algorithms to determine the 
target parameters [6], [7]. The performance of this kind of methods is superior to that of the indirect approaches at the low SNRs. Nevertheless, they demand huge data communication and computational costs, which brings a very high processing complexity. Beyond that, some new schemes are proposed in terms of the direct localization. In [29], a sparse modeling based approach is developed to estimate the parameters of multiple targets. In [30], the problem of joint detection and localization multiple targets is discussed, and two suboptimal and low complexity solutions are proposed to tackle this issue. The work [31] designs a factor graph approach for target localization, which can reduce the computational complexity [32] and the communication cost effectively.

In this paper, we propose a novel solution for multi-target counting and localization in the distributed MIMO radar. In order to figure out the localization accuracy for multiple targets based on the distributed MIMO radar, we first investigate the theoretical lower bound of the multi-target localization in terms of the considered discrete time signal model. The bound is composed of two parts, i.e., the Cramér-Rao lower bound (CRLB) and the sampling lower bound (SLB). The CRLB is related to the SNR, and the lower bound is specified by it at the low SNR regime. On the other side, the localization accuracy is bounded by the SLB when the SNR is high, which is independent of the SNR, but associated with the sampling rate. Afterwards, an energy modeling and compressive sensing based scheme is designed to cope with the multi-target counting and localization problem. The main insight of the scheme is that the energy value of a given position, which is obtained by the multi-path accumulation, is dependent on the parameters of the targets as well as the geometric distribution of the targets and the antennas. Therefore, for a fixed layout of the distributed MIMO radar, we can estimate the number and the locations of the targets from the energy values of numerous points with known coordinates. In addition, considering that the targets are sparsely distributed, the compressive sensing theory is employed to obtain the interested information based on limited measurements of the energy values. This means that the data communication loads among the antennas and the data fusion center can be reduced significantly. To recover the multi-target information efficiently, we further develop a low complexity greedy pursuit algorithm, which combines the well-known matching pursuit (MP) algorithm [33] and the similarity evaluation strategy. The effectiveness and robustness of the proposed method is evaluated via the simulations. It is worth mentioning that the work [29] also involves the technique of compressive sensing recovery, however, its inherent idea is completely different from ours. Specifically, the method in [29] considers the sparsity of each transmitter-receiver path independently, which means that a high single path SNR is necessary to ensure the effective recovery. Moreover, the issue of target counting is not discussed in that work.

In summary, the main contributions of this paper include threefold:

1) We provide the theoretical lower bound for the multitarget localization scenario under the discrete time signal model. It is a combination of the CRLB and the SLB, and consequently is associated with the SNR and the sampling rate.

2) We develop a novel framework in terms of the problem of multi-target counting and localization. It takes advantage of the energy modeling and the compressive sensing, and can significantly alleviate the data communication burden compared with the conventional direct localization methods.

3) An efficient and simple sparse vector recovery algorithm is proposed for the above framework. It utilizes the MP algorithm as the main body, and integrates the similarity evaluation strategy, which can produce superior performance on multi-target counting and localization.

The rest of this paper is arranged as follows. Section II describes the considered signal model. Section III discusses the lower bound of the multi-target localization accuracy. The solution of the multi-target counting and localization problem is presented in Section IV. The simulation results are given in Section V. Finally, the conclusion is drawn in Section VI

Notation: In this paper, the superscripts $(\cdot)^{T},(\cdot)^{*}$, and $(\cdot)^{H}$ represent the transpose, conjugate, and the conjugate transpose, respectively. $\mathbb{C}$ and $\mathbb{R}$ denote the complex number field and the real number field, respectively. $|\cdot|$ denotes the absolute value for the real number and the complex magnitude for the complex number. $\|\cdot\|$ denotes the Euclidean norm. $\lfloor\cdot 7$ indicates rounding to the nearest integer. $\mathbf{I}_{a}$ represents the identity matrix with the size of $a \times a$, and $\mathbf{0}_{a \times b}$ represents the zero vector/matrix with the size of $a \times b$. $\mathbb{E}[\cdot]$ stands for the expectation. $\operatorname{tr}(\cdot)$ and $\operatorname{det}(\cdot)$ denote the trace and the determinant of the matrix, respectively. $\operatorname{diag}(\cdot)$ represents the diagonal matrix composed of the elements in parentheses, and blkdiag $(\cdot)$ denotes the block diagonal matrix formed by the matrices in parentheses.

\section{Signal Model}

We consider a distributed MIMO radar system with $M$ transmitters and $N$ receivers in a 2-dimensional (2-D) space. The antennas are located arbitrarily in a given area with known coordinates. For the $m$ th transmitter, the position is denoted as $\mathbf{T}_{m}=\left[x_{m}^{t}, y_{m}^{t}\right]^{T}$, while it is $\mathbf{R}_{n}=\left[x_{n}^{r}, y_{n}^{r}\right]^{T}$ for the $n$th receiver. Let $\sqrt{E} s_{m}(t)$ denote the lowpass equivalent form of the signal transmitted by the $m$ th transmitter, where $E$ is the transmitted energy, assume to be consistent for different transmitters, and $s_{m}(t)$ represents the normalized waveform, i.e., $\int_{-\infty}^{+\infty}\left|s_{m}(t)\right|^{2} d t=1$. We further assume that signals from different transmitters are orthogonal, and they approximately maintain orthogonality for the allowable time delay $\tau$, that is,

$$
\int_{-\infty}^{+\infty} s_{m_{1}}(t) s_{m_{2}}^{*}(t-\tau) d t \approx 0 \quad \text { if } m_{1} \neq m_{2} .
$$

Hence, different transmitted signals can be separately at each receiver via the corresponding matched filters [7]. The system observes a specific region of interest, which is approximated by a circle $\Theta$ for ease of discussion. Consider a scenario with $K \geq 1$ point targets distributed in the surveillance region $\boldsymbol{\Theta}$, all the targets are stationary during the signal processing period, and thus the Doppler effect is negligible [31]. The 
position of the $k$ th target is expressed as $\mathbf{u}_{k}=\left[x_{k}^{u}, y_{k}^{u}\right]^{T}$, which is treated as a deterministic unknown.

At the $n$th receiver, the baseband form of the received signal is the echo superposition of $M$ transmitted signals reflected by $K$ targets, which is written as

$$
r_{n}(t)=\sqrt{E} \sum_{m=1}^{M} \sum_{k=1}^{K} \alpha_{m n k} s_{m}\left(t-\tau_{m n k}\right)+\omega_{n}(t)
$$

where $\alpha_{m n k}$ denotes the complex-valued reflection coefficient with regard to the $m n$th transmitter-receiver path and the $k$ th target, including the comprehensive influence of the target radar cross section (RCS), the path loss, the antenna gains, etc [34]. Herein, it is modeled as a complex Gaussian distribution with $\alpha_{m n k} \sim \mathcal{C N}\left(0, \sigma_{m n k}^{2}\right)$, and is constant within the signal period. Besides, the value of $\left|\alpha_{m n k}\right|^{2}$ is related to the distance between the target and the antennas, i.e.,

$$
\left|\alpha_{m n k}\right|^{2}=\frac{\left|\eta_{k}\right|^{2}}{\left\|\mathbf{u}_{k}-\mathbf{T}_{m}\right\|^{2}\left\|\mathbf{u}_{k}-\mathbf{R}_{n}\right\|^{2}}=\frac{\left|\eta_{k}\right|^{2}}{d_{t m k}^{2} d_{r n k}^{2}}
$$

where $\eta_{k}$ also obeys the complex Gaussian distribution with $\eta_{k} \sim \mathcal{C N}\left(0, \sigma_{k}^{2}\right)$. This implies that $\sigma_{m n k}^{2}=\sigma_{k}^{2} /\left(d_{t m k}^{2} d_{r n k}^{2}\right)$. The term $\tau_{m n k}$ represents the time delay associated with positions of the $m$ th transmitter, the $n$th receiver, and the $k$ th target, which is computed as

$$
\tau_{m n k}=\frac{d_{t m k}+d_{r n k}}{c}
$$

where $c$ is the speed of light. The term $\omega_{n}(t)$ is the additive noise, and is modeled as a white complex Gaussian random process with $\mathbb{E}\left[\omega_{n}\left(t_{1}\right)\right]=0, \mathbb{E}\left[\omega_{n}\left(t_{1}\right) \omega_{n}^{*}\left(t_{2}\right)\right]=$ $\sigma_{\omega}^{2} \delta\left(t_{1}-t_{2}\right)$, and $\mathbb{E}\left[\omega_{n_{1}}\left(t_{1}\right) \omega_{n_{2}}^{*}\left(t_{2}\right)\right]=0$ if $n_{1} \neq n_{2}$, where $\delta(t)$ represents the unit impulse function [6].

It is considered that the continuous signals are sampled as the discrete signals at the sampling rate $f_{s}$, and then (2) is recast as

$$
\begin{aligned}
& r_{n}[i]=\sqrt{E} \sum_{m=1}^{M} \sum_{k=1}^{K} \alpha_{m n k} s_{m}\left[i-i_{m n k}\right]+\omega_{n}[i] \\
& i=1,2, \ldots, I
\end{aligned}
$$

where $i=\left\lfloor t \times f_{s}\right\rceil, i_{m n k}=\left\lfloor\tau_{m n k} \times f_{s}\right\rceil$, and $I=\left\lfloor\mathcal{T} \times f_{s}\right\rceil$ denote the sampling indices at different times, and $\mathcal{T}$ is the signal period. In order to ensure the normalization of $s_{m}\left[i-i_{m n k}\right]$, that is, $\sum_{i}^{I}\left|s_{m}\left[i-i_{m n k}\right]\right|^{2}=1$, we have $s_{m}\left[i-i_{m n k}\right]=\left(1 / \sqrt{f_{s}}\right) s_{m}\left(t-\tau_{m n k}\right)$. Besides, for the convenience of the analysis and derivation, we present the following definitions.

$$
\begin{aligned}
& \mathbf{r}_{n}=\left[r_{n}[1], \ldots, r_{I}[I]\right]^{T}, \mathbf{r}=\left[\mathbf{r}_{1}^{T}, \ldots, \mathbf{r}_{N}^{T}\right]^{T}, \\
& \boldsymbol{\alpha}_{m n}=\left[\alpha_{m n 1}, \ldots, \alpha_{m n K}\right]^{T}, \boldsymbol{\alpha}_{n}=\left[\boldsymbol{\alpha}_{1 n}^{T}, \ldots, \boldsymbol{\alpha}_{M n}^{T}\right]^{T}, \\
& \boldsymbol{\alpha}=\left[\boldsymbol{\alpha}_{1}^{T}, \ldots, \boldsymbol{\alpha}_{N}^{T}\right]^{T}, \\
& \mathbf{s}_{m n k}=\left[s_{m}\left[1-i_{m n k}\right], \ldots, s_{m}\left[I-i_{m n k}\right]\right]^{T} \\
& \mathbf{S}_{m n}=\left[\mathbf{s}_{m n 1}, \ldots, \mathbf{s}_{m n K}\right], \mathbf{S}_{n}=\left[\mathbf{S}_{1 n}, \ldots, \mathbf{S}_{M n}\right] \\
& \mathbf{S}=\operatorname{blkdiag}\left(\mathbf{S}_{1}, \ldots, \mathbf{S}_{N}\right), \\
& \boldsymbol{\omega}_{n}=\left[\omega_{n}[1], \ldots, \omega_{n}[I]\right]^{T}, \boldsymbol{\omega}=\left[\boldsymbol{\omega}_{1}^{T}, \ldots, \boldsymbol{\omega}_{N}^{T}\right]^{T} .
\end{aligned}
$$

Therefore, the ensemble form of all the received signals can be written as

$$
\mathbf{r}=\sqrt{E} \mathbf{S} \boldsymbol{\alpha}+\boldsymbol{\omega} .
$$

Based on the signal model described above, our purpose is to estimate the number as well as the positions of the multiple targets from the received signals directly.

\section{Bound ANALysis FOR Multi-TARget LOCALIZATION}

In this section, the multi-target localization accuracy under the considered scenario is evaluated. We first derive the CRLB of the signal model presented in Section III Moreover, since the signal model is in discrete time, we also analyze the corresponding SLB determined by the sampling interval $1 / f_{s}$. The combination of these two lower bounds is regarded as the theoretical bound of the multi-target localization.

\section{A. $C R L B$}

The CRLB indicates a lower bound on the variance of any unbiased estimator [35]. Define the unknown parameter vector $\boldsymbol{\theta}=\left[\mathbf{u}_{1}^{T}, \ldots, \mathbf{u}_{K}^{T}\right]^{T}$, then the CRLB matrix of $\boldsymbol{\theta}$ can be expressed as [5], [36]

$$
\begin{aligned}
& \mathbf{C}_{\mathrm{CRLB}}(\boldsymbol{\theta})=[\mathbf{J}(\boldsymbol{\theta})]^{-1} \\
& =\mathbb{E}\left[\left(\frac{\partial \ln p(\mathbf{r} \mid \boldsymbol{\theta})}{\partial \boldsymbol{\theta}}\right)\left(\frac{\partial \ln p(\mathbf{r} \mid \boldsymbol{\theta})}{\partial \boldsymbol{\theta}}\right)^{T}\right]^{-1}
\end{aligned}
$$

where $\mathbf{J}(\boldsymbol{\theta})$ represents the Fisher information matrix (FIM) of $\boldsymbol{\theta}$, and $p(\mathbf{r} \mid \boldsymbol{\theta})$ is the probability density function (PDF) of $\mathbf{r}$. According to the expression of (7),p $(\mathbf{r} \mid \boldsymbol{\theta})$ is written as

$$
p(\mathbf{r} \mid \boldsymbol{\theta})=\frac{1}{\pi^{N I} \operatorname{det}(\boldsymbol{\Sigma})} e^{-\mathbf{r}^{H} \boldsymbol{\Sigma}^{-1} \mathbf{r}}
$$

where $\boldsymbol{\Sigma}$ stands for the covariance matrix of $\mathbf{r}$, that is,

$$
\begin{aligned}
& \boldsymbol{\Sigma}=\mathbb{E}\left[(\sqrt{E} \mathbf{S} \boldsymbol{\alpha}+\boldsymbol{\omega})(\sqrt{E} \mathbf{S} \boldsymbol{\alpha}+\boldsymbol{\omega})^{H}\right] \\
& =E \mathbf{S} \boldsymbol{\Sigma}_{\alpha} \mathbf{S}^{H}+\boldsymbol{\Sigma}_{\omega} \\
& =\operatorname{blkdiag}\left(E \mathbf{S}_{1} \boldsymbol{\Sigma}_{\alpha_{1}} \mathbf{S}_{1}^{H}+\boldsymbol{\Sigma}_{\omega_{1}}, \ldots, E \mathbf{S}_{N} \boldsymbol{\Sigma}_{\alpha_{N}} \mathbf{S}_{N}^{H}+\boldsymbol{\Sigma}_{\omega_{N}}\right)
\end{aligned}
$$

where

$$
\begin{aligned}
& \boldsymbol{\Sigma}_{\alpha_{n}}=\mathbb{E}\left[\boldsymbol{\alpha}_{\boldsymbol{n}} \boldsymbol{\alpha}_{n}^{H}\right]=\operatorname{diag}\left(\sigma_{1 n 1}^{2}, \ldots, \sigma_{M n K}^{2}\right) \\
& \boldsymbol{\Sigma}_{\omega_{n}}=\mathbb{E}\left[\boldsymbol{\omega}_{\boldsymbol{n}} \boldsymbol{\omega}_{\boldsymbol{n}}^{H}\right]=\sigma_{\omega}^{2} \mathbf{I}_{I} \\
& \boldsymbol{\Sigma}_{\alpha}=\mathbb{E}\left[\boldsymbol{\alpha} \boldsymbol{\alpha}^{H}\right]=\operatorname{blkdiag}\left(\boldsymbol{\Sigma}_{\alpha_{1}}, \ldots, \boldsymbol{\Sigma}_{\alpha_{N}}\right) \\
& \boldsymbol{\Sigma}_{\omega}=\mathbb{E}\left[\boldsymbol{\omega} \boldsymbol{\omega}^{H}\right]=\sigma_{\omega}^{2} \mathbf{I}_{N I}
\end{aligned}
$$

Considering that the received signals contained in (7) are functions with respect to the time delay $\tau_{m n k}$, we can rewrite the FIM by using the chain rule to facilitate the derivation [5], [37], i.e.,

$$
\mathbf{J}(\boldsymbol{\theta})=\left(\frac{\partial \boldsymbol{\zeta}}{\partial \boldsymbol{\theta}}\right) \mathbf{J}(\boldsymbol{\zeta})\left(\frac{\partial \boldsymbol{\zeta}}{\partial \boldsymbol{\theta}}\right)^{T}
$$

where $\zeta$ is the vector composed of the time delays of all the paths and the targets, which is given as $\boldsymbol{\zeta}=\left[\boldsymbol{\tau}_{1}^{T}, \ldots, \boldsymbol{\tau}_{N}^{T}\right]^{T}$, with $\boldsymbol{\tau}_{n}=\left[\boldsymbol{\tau}_{1 n}^{T}, \ldots, \boldsymbol{\tau}_{M n}^{T}\right]^{T}$ and $\boldsymbol{\tau}_{m n}^{T}=\left[\tau_{m n 1}, \ldots, \tau_{m n K}\right]^{T}$. 
The term $\mathbf{J}(\boldsymbol{\zeta})$ denotes the FIM of the vector $\boldsymbol{\zeta}$, and the elements in $\partial \boldsymbol{\zeta} / \partial \boldsymbol{\theta}$ are arranged as

$$
\frac{\partial \boldsymbol{\zeta}}{\partial \boldsymbol{\theta}}=\left[\begin{array}{cccc}
\frac{\partial \tau_{111}}{\partial x_{1}^{u}} & \frac{\partial \tau_{112}}{\partial x_{1}^{u}} & \ldots & \frac{\partial \tau_{M N K}}{\partial x_{1}^{u}} \\
\frac{\partial \tau_{111}}{\partial y_{1}^{u}} & \frac{\partial \tau_{112}}{\partial y_{1}^{u}} & \ldots & \frac{\partial \tau_{M N K}}{\partial y_{1}^{u}} \\
\vdots & \vdots & \ddots & \vdots \\
\frac{\partial \tau_{111}}{\partial y_{K}^{u}} & \frac{\partial \tau_{112}}{\partial y_{K}^{u}} & \ldots & \frac{\partial \tau_{M N K}}{\partial y_{K}^{u}}
\end{array}\right]_{2 K \times M N K}
$$

while the expression of the partial derivative in the matrix (13) can be further written as

$$
\begin{aligned}
& \frac{\partial \tau_{m n k}}{\partial x_{k}^{u}}=\frac{1}{c}\left[\frac{\left(x_{k}^{u}-x_{m}^{t}\right)}{d_{t m k}}+\frac{\left(x_{k}^{u}-x_{n}^{r}\right)}{d_{r n k}}\right] \\
& \frac{\partial \tau_{m n k}}{\partial y_{k}^{u}}=\frac{1}{c}\left[\frac{\left(y_{k}^{u}-y_{m}^{t}\right)}{d_{t m k}}+\frac{\left(y_{k}^{u}-y_{n}^{r}\right)}{d_{r n k}}\right] .
\end{aligned}
$$

Next, we compute the FIM $\mathbf{J}(\boldsymbol{\zeta})$. Based on the PDF (9), the elements in $\mathbf{J}(\boldsymbol{\zeta})$ can be written as [35]

$$
[\mathbf{J}(\boldsymbol{\zeta})]_{q, q^{\prime}}=\operatorname{tr}\left(\boldsymbol{\Sigma}^{-1} \frac{\partial \boldsymbol{\Sigma}}{\partial \zeta_{q}} \boldsymbol{\Sigma}^{-1} \frac{\partial \boldsymbol{\Sigma}}{\partial \zeta_{q^{\prime}}}\right)
$$

where $q, q^{\prime}=1,2, \ldots, M N K$, and the derivation of 15 is presented in Appendix A By substituting (13), 114, 500, and (51) into (12), we can acquire the FIM $\mathbf{J}(\boldsymbol{\theta})$, and the CRLB matrix $\mathbf{C}_{\mathrm{CRLB}}(\boldsymbol{\theta})$ can be generated accordingly by inverting the matrix $\mathbf{J}(\boldsymbol{\theta})$. For the $k$ th target, the root CRLB is given by

$$
\begin{aligned}
& \operatorname{RCRLB}\left(\mathbf{u}_{k}\right) \\
& =\sqrt{\left[\mathbf{C}_{\mathrm{CRLB}}(\boldsymbol{\theta})\right]_{2 k-1,2 k-1}+\left[\mathbf{C}_{\mathrm{CRLB}}(\boldsymbol{\theta})\right]_{2 k, 2 k}} .
\end{aligned}
$$

It is worth mentioning that 16 neglects the influence of the discrete sampling, and this point will be discussed in the next subsection.

\section{B. $S L B$}

As described in Section [I] for a given time delay $\tau_{m n k}$, we convert it into the discrete index $i_{m n k}$ by multiplying the sampling rate $f_{s}$ and then rounding the value. In other words, $\tau_{m n k}$ is separated into $i_{m n k}$ segments with the unit of the sampling interval $1 / f_{s}$. Herein, the discrete sampling error is produced. In order to analyze the localization performance boundary with the existence of the sampling error, we need to consider the optimal case of the time delay estimation, that is, all the time delays are sampled correctly. Let $d_{m n k}$ denote the measured bistatic range with regard to the $m n$th transmitterreceiver path and the $k$ th target under the optimal case, and we can model it as

$$
d_{m n k}=c \tau_{m n k}+\varepsilon=d_{t m k}+d_{r n k}+\varepsilon
$$

where $\varepsilon$ represents the measurement error. For the purpose of derivation, $\varepsilon$ is assumed to obey the Gaussian distribution with $\varepsilon \sim \mathcal{N}\left(0, \sigma_{\varepsilon}^{2}\right)$. How to determine the variance $\sigma_{\varepsilon}^{2}$ is an interesting issue. It is natural that the value of $\varepsilon$ should be in the interval $\left[-c / 2 f_{s}, c / 2 f_{s}\right]$ with a high probability for the optimal case, which can be expressed as

$$
\begin{aligned}
& p\left(-\frac{c}{2 f_{s}} \leq \varepsilon \leq \frac{c}{2 f_{s}}\right) \\
& =\frac{1}{2}\left(\operatorname{erf}\left(\frac{c}{2 \sqrt{2} f_{s} \sigma_{\varepsilon}}\right)-\operatorname{erf}\left(-\frac{c}{2 \sqrt{2} f_{s} \sigma_{\varepsilon}}\right)\right) \approx \gamma
\end{aligned}
$$

where $\operatorname{erf}(\cdot)$ is the error function [38], and $\gamma$ denotes the given probability. Without loss of generality, we set $\gamma=0.9$, which yields that $c / 2 f_{s}=1.65 \sigma_{\varepsilon}$, and thus we have $\sigma_{\varepsilon}^{2}=$ $\left(c / 3.3 f_{s}\right)^{2}$.

Next, we derive the localization lower bound based on the relevant CRLB. For the $k$ th target, the joint PDF of the bistatic ranges is given as

$$
\begin{aligned}
& p\left(\left\{d_{m n k}\right\}_{m=1, n=1}^{M, N} \mid \mathbf{u}_{k}\right) \\
& =\prod_{m=1}^{M} \prod_{n=1}^{N} \frac{1}{\sqrt{2 \pi \sigma_{\varepsilon}^{2}}} e^{-\frac{\left(d_{m n k}-d_{t m k}-d_{r n k}\right)^{2}}{2 \sigma_{\varepsilon}^{2}}}
\end{aligned}
$$

and the FIM of $\mathbf{u}_{k}$ is therefore written as

$$
\mathbf{J}\left(\mathbf{u}_{k}\right)=\left[\begin{array}{ll}
\mathbb{E}\left[\frac{\partial \ln p}{\partial x_{k}^{u}} \frac{\partial \ln p}{\partial x_{k}^{u}}\right. \\
\mathbb{E}\left[\begin{array}{ll}
\frac{\partial \ln p}{\partial y_{k}^{u}} \frac{\partial \ln p}{\partial x_{k}^{u}}
\end{array}\right] & \mathbb{E}\left[\begin{array}{l}
\frac{\partial \ln p}{\partial x_{k}^{u}} \frac{\partial \ln p}{\partial y_{k}^{u}} \\
\frac{\partial \ln p}{\partial y_{k}^{u}} \frac{\partial \ln p}{\partial y_{k}^{u}}
\end{array}\right]
\end{array}\right]
$$

and each element in 20 is given as

$$
\begin{aligned}
& \mathbb{E}\left[\frac{\partial \ln p}{\partial x_{k}^{u}} \frac{\partial \ln p}{\partial x_{k}^{u}}\right] \\
& =\frac{1}{\sigma_{\varepsilon}^{2}} \sum_{m=1}^{M} \sum_{n=1}^{N}\left(\frac{x_{k}^{u}-x_{m}^{t}}{d_{t m k}}+\frac{x_{k}^{u}-x_{n}^{r}}{d_{r n k}}\right)^{2} \\
& \mathbb{E}\left[\frac{\partial \ln p}{\partial x_{k}^{u}} \frac{\partial \ln p}{\partial y_{k}^{u}}\right]=\mathbb{E}\left[\frac{\partial \ln p}{\partial y_{k}^{u}} \frac{\partial \ln p}{\partial x_{k}^{u}}\right] \\
& =\frac{1}{\sigma_{\varepsilon}^{2}} \sum_{m=1}^{M} \sum_{n=1}^{N}\left(\frac{x_{k}^{u}-x_{m}^{t}}{d_{t m k}}+\frac{x_{k}^{u}-x_{n}^{r}}{d_{r n k}}\right) \\
& \times\left(\frac{y_{k}^{u}-y_{m}^{t}}{d_{t m k}}+\frac{y_{k}^{u}-y_{n}^{r}}{d_{r n k}}\right) \\
& \mathbb{E}\left[\frac{\partial \ln p}{\partial y_{k}^{u}} \frac{\partial \ln p}{\partial y_{k}^{u}}\right] \\
& =\frac{1}{\sigma_{\varepsilon}^{2}} \sum_{m=1}^{M} \sum_{n=1}^{N}\left(\frac{y_{k}^{u}-y_{m}^{t}}{d_{t m k}}+\frac{y_{k}^{u}-y_{n}^{r}}{d_{r n k}}\right)^{2} .
\end{aligned}
$$

Then the CRLB matrix $\mathbf{C}_{\mathrm{CRLB}}\left(\mathbf{u}_{k}\right)$ can be obtained by computing $\left[\mathbf{J}\left(\mathbf{u}_{k}\right)\right]^{-1}$. Similar to the definition in $(16$, the root SLB of the target $\mathbf{u}_{k}$ is written as

$$
\operatorname{RSLB}\left(\mathbf{u}_{k}\right)=\sqrt{\operatorname{tr}\left(\mathbf{C}_{\mathrm{CRLB}}\left(\mathbf{u}_{k}\right)\right)} .
$$

Combining (16) and 24), the lower bound of the localization root mean square error (RMSE) for the $k$ th target can be expressed as

$$
\operatorname{RMSE}\left(\mathbf{u}_{k}\right) \geq \max \left(\operatorname{RCRLB}\left(\mathbf{u}_{k}\right), \operatorname{RSLB}\left(\mathbf{u}_{k}\right)\right) .
$$

\section{An Example}

We present a simple example to describe the bound intuitively. For the convenience of discussion, we adopt the scenario given in Section $\mathrm{V}-\mathrm{A}$, which includes 4 transmitters, 6 receivers, and 3 targets. In addition, the signal we used is also specified in Section $\mathrm{V}-\mathrm{A}$, except that three sampling rate cases with $8 \mathrm{MHz}, 10 \mathrm{MHz}$, and $12 \mathrm{MHz}$ are considered. Fig. 1 gives the RCRLB and RSLB versus SNR (defined in (38) ) with different sampling rate cases for the three targets. It is seen that the lower bound of the localization accuracy for 


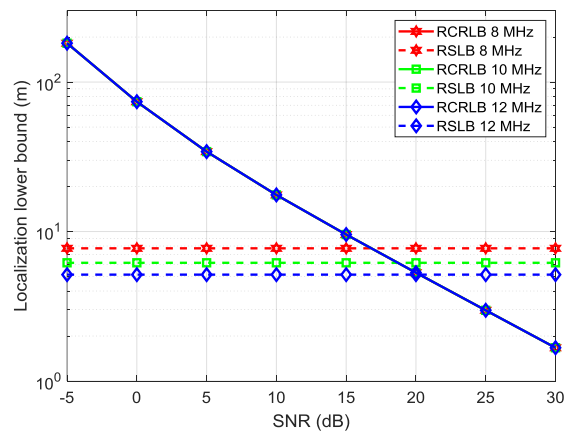

(a)

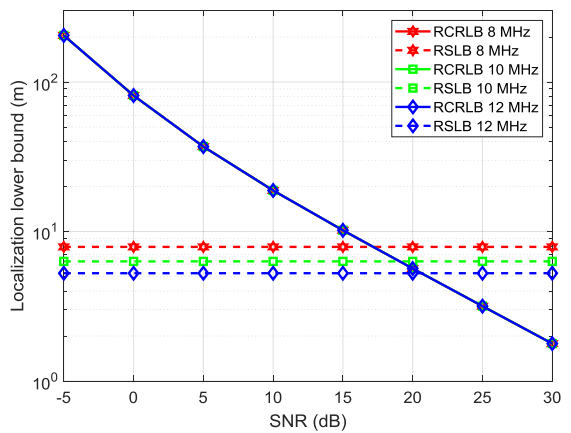

(b)

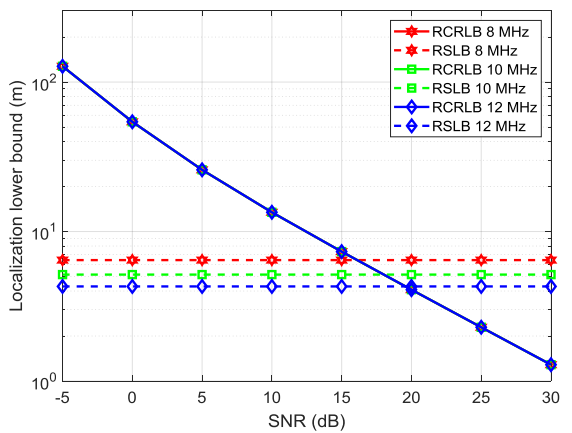

(c)

Fig. 1. An example of RCRLB and RSLB versus SNR with different sampling rate cases. (a) The 1st target. (b) The 2nd target. (c) The 3rd target.

each target is dominated by the RCRLB at the low SNR region (e.g., SNR $\leq 15 \mathrm{~dB}$ ), while the RSLB is the decisive factor in the case of the high SNR. The RCRLB decreases with the increase of the SNR, and the RSLB decreases with the rise of the sampling rate. Moreover, the effect of the sampling rate on the RCRLB is negligible, and the RSLB is independent of the SNR. We can notice that the phenomenon presented in the example is consistent with the theoretical analysis above.

\section{Solution of Multi-Target Counting And LOCALIZATION}

In this section, we elaborate on the solution of the multitarget counting and localization with the distributed MIMO radar. The framework is based on the compressive sensing theory, and is mainly composed of two schemes. 1) A dictionary matrix construction method based on energy modeling; and 2) an efficient sparse vector recovery strategy. Before proceeding, we first present a schematic flowchart of the proposal, as shown in Fig. 2.

\section{A. Preliminaries of Compressive Sensing}

The compressive sensing theory states that it is possible to reconstruct a sparse signal from much fewer measurements than the conventional methods. Assume that $\Psi=$ $\left[\boldsymbol{\psi}_{1}, \ldots, \boldsymbol{\psi}_{G}\right] \in \mathbb{R}^{G \times G}$ is a basis matrix, and each column $\boldsymbol{\psi}_{g} \in \mathbb{R}^{G \times 1}$ in $\boldsymbol{\Psi}$ denotes a basis vector. For a given signal $\mathbf{x} \in \mathbb{R}^{G \times 1}$, it can be expressed as [39]

$$
\mathbf{x}=\boldsymbol{\Psi} \mathbf{h}=\sum_{g=1}^{G} h_{g} \boldsymbol{\psi}_{g}
$$

where $\mathbf{h}=\left[h_{1}, \ldots, h_{G}\right]^{T} \in \mathbb{R}^{G \times 1}$ is a $K$-sparse coefficient vector, that is, $\|\mathbf{h}\|_{0}=K$ and $K \ll G$. According to the compressive sensing theory, we can recover $\mathbf{h}$ by using a small number of measurements that much less than $G$, i.e.,

$$
\mathbf{y}=\boldsymbol{\Phi} \mathbf{x}+\mathbf{z}=\underbrace{\Phi \Psi}_{\mathbf{A}} \mathbf{h}+\mathbf{z}
$$

where $\mathbf{y} \in \mathbb{R}^{L \times 1} \quad(K<L \ll G)$ stands for the measurement vector, $\boldsymbol{\Phi} \in \mathbb{R}^{L \times G}$ is the measurement matrix, and $\mathbf{z} \in \mathbb{R}^{L \times 1}$ is the noise. It has been shown that the number of measurements to ensure the effective recovery is $L=$ $\mathcal{O}(K \log (G / K))$ [40].

Numerous approaches have been developed to solve the signal reconstruction problem, which can be roughly divided into four categories [41]-[43]. 1) Greedy pursuits. This kind of methods recover the signal in an iterative way, and identify the local optimal solution in each iteration. Representative examples include the MP [33], the orthogonal matching pursuit (OMP) [44], and the compressive sampling matching pursuit (CoSaMP) [43]. 2) Convex relaxation. This kind of methods realize the signal reconstruction by solving a convex optimization problem. Such as the interior-point methods [45], the least absolute shrinkage and selection operator (LASSO) [46], and the gradient projection algorithms [47]. 3) Nonconvex optimization. These methods convert the problem to some related nonconvex optimization problems, e.g., the $\ell_{p}$ norm minimization with $0<p<1$ [48]. 4) Bayesian compressive sensing. These techniques assume a sparseness prior for the unknown signal, and solve the problem based on a maximum a posteriori (MAP) estimation [49].

\section{B. Energy Modeling Based Dictionary Matrix Construction}

Suppose that the surveillance region $\Theta$ is broken into a large quantity of girds with the same size. Let $G$ and $\Delta d \times \Delta d$ be the number of the girds and the grid size, while $\mathbf{P}_{g}=\left[x_{g}^{p}, y_{g}^{p}\right]^{T}$ represents the center position of the $g$ th $(g=1, \ldots, G)$ grid. For a given grid $\mathbf{P}_{g}$, we define the corresponding noise-free energy as

$$
\begin{aligned}
& \Lambda\left(\mathbf{P}_{g}\right) \\
& =\frac{1}{M N} \sum_{m=1}^{M} \sum_{n=1}^{N} \mid \int_{-\infty}^{+\infty} \sum_{m=1}^{M} \sum_{k=1}^{K} \alpha_{m n k} s_{m}\left(t-\tau_{m n k}\right) \\
& \times\left. s_{m}^{*}\left(t-\tau_{m n}\left(\mathbf{P}_{g}\right)\right) d t\right|^{2} \\
& \approx \frac{1}{M N} \sum_{m=1}^{M} \sum_{n=1}^{N} \sum_{k=1}^{K}\left|\alpha_{m n k}\right|^{2} \mid \int_{-\infty}^{+\infty} s_{m}\left(t-\tau_{m n k}\right) \\
& \times\left. s_{m}^{*}\left(t-\tau_{m n}\left(\mathbf{P}_{g}\right)\right) d t\right|^{2} \\
& =\frac{1}{M N} \sum_{k=1}^{K}\left|\eta_{k}\right|^{2} \sum_{m=1}^{M} \sum_{n=1}^{N} \frac{1}{d_{t m}^{k 2} d_{r n}^{k 2}} \mid \int_{-\infty}^{+\infty} s_{m}\left(t-\tau_{m n k}\right) \\
& \times\left. s_{m}^{*}\left(t-\tau_{m n}\left(\mathbf{P}_{g}\right)\right) d t\right|^{2}
\end{aligned}
$$




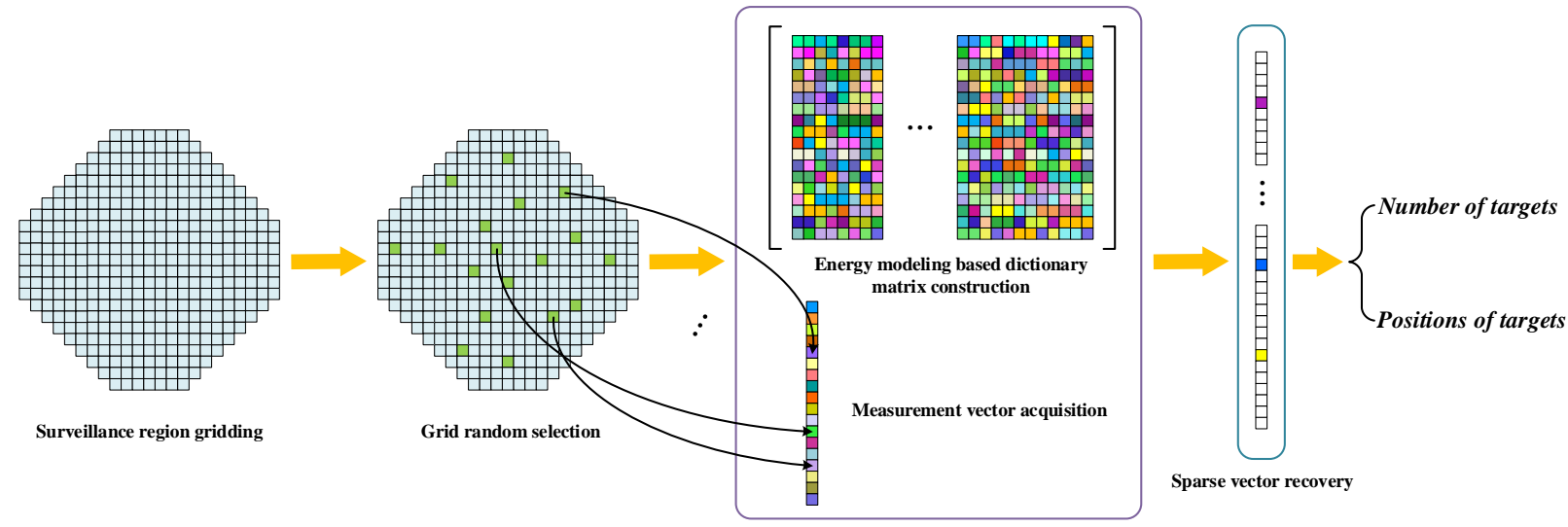

Fig. 2. Schematic flowchart of the proposed multi-target counting and localization solution.

where $\tau_{m n}\left(\mathbf{P}_{g}\right)$ is the time delay with regard to the $m$ th transmitter, the $n$th receiver, and the grid $\mathbf{P}_{g}$, i.e.,

$$
\tau_{m n}\left(\mathbf{P}_{g}\right)=\frac{\left\|\mathbf{P}_{g}-\mathbf{T}_{m}\right\|+\left\|\mathbf{P}_{g}-\mathbf{R}_{n}\right\|}{c}
$$

In fact, the energy $\Lambda\left(\mathbf{P}_{g}\right)$ in 28$)$ can be interpreted as the weighted sum of the magnitude squares of the $M N K$ matched filter outputs, and scaling according to the coefficient $1 / M N$. It is worth noting that the second approximate relationship in (28) invokes three assumptions. 1) The orthogonal property of the transmitted signals, which has been stated in Section III. 2) the magnitude of the mnkth matched filter output possesses the narrow-pulse property, and it reaches the peak when $\tau_{m n}\left(\mathbf{P}_{g}\right)=\tau_{m n k}$; and 3) there is at most one target in a given gird. Specifically, by using the assumption 1, we have

$$
\begin{aligned}
& \left|\int_{-\infty}^{+\infty} \sum_{m=1}^{M} \sum_{k=1}^{K} \alpha_{m n k} s_{m}\left(t-\tau_{m n k}\right) s_{m}^{*}\left(t-\tau_{m n}\left(\mathbf{P}_{g}\right)\right) d t\right|^{2} \\
& \approx\left|\sum_{k=1}^{K} \alpha_{m n k} \int_{-\infty}^{+\infty} s_{m}\left(t-\tau_{m n k}\right) s_{m}^{*}\left(t-\tau_{m n}\left(\mathbf{P}_{g}\right)\right) d t\right|^{2}
\end{aligned}
$$

then expanding the expression of 30 and using the assumptions 2 and 3 , we can obtain that

$$
\begin{aligned}
& \left(\alpha_{m n k_{1}} \int_{-\infty}^{+\infty} s_{m}\left(t-\tau_{m n k_{1}}\right) s_{m}^{*}\left(t-\tau_{m n}\left(\mathbf{P}_{g}\right)\right) d t\right) \\
& \times\left(\alpha_{m n k_{2}} \int_{-\infty}^{+\infty} s_{m}\left(t-\tau_{m n k_{2}}\right) s_{m}^{*}\left(t-\tau_{m n}\left(\mathbf{P}_{g}\right)\right) d t\right)^{*} \\
& \approx 0 \quad \text { if } k_{1} \neq k_{2} .
\end{aligned}
$$

On the basis of (30) and (31), the approximate relationship in (28) holds.

Apparently, the energy $\Lambda\left(\mathbf{P}_{g}\right)$ is an effective indicator that can be used to determine whether there exists a target in the grid $\mathbf{P}_{g}$. According to the characteristics of the matched filter output, the energy value of the grid where the target is located is the local maximum. Furthermore, $\Lambda\left(\mathbf{P}_{g}\right)$ also reflects the relative geometric relationship between the gird $\mathbf{P}_{g}$ and each target. In other words, different grids have their specific energy values, which are related to the positions of the targets. Therefore, in order to determine the number and the positions of the multiple targets in the surveillance region, we can first acquire the energy values of all the grids, form a vector $\mathbf{x}^{\Lambda}=\left[\Lambda\left(\mathbf{P}_{1}\right), \ldots, \Lambda\left(\mathbf{P}_{G}\right)\right]^{T}$; and then construct a $G \times G$ matrix $\Psi^{\Lambda}$, which is expressed in (32), at the bottom of the next page, where $d_{t m}^{P_{g}}=\left\|\mathbf{P}_{g}-\mathbf{T}_{m}\right\|$ and $d_{r n}^{P_{g}}=\left\|\mathbf{P}_{g}-\mathbf{R}_{n}\right\|$. Based on the definition in (28), the vector $\mathrm{x}^{\Lambda}$ can be represented by the linear weighted superposition of $K$ columns in the matrix $\Psi^{\Lambda}$, that is,

$$
\mathbf{x}^{\Lambda}=\Psi^{\Lambda} \mathbf{h}^{\Lambda}
$$

where $\mathbf{h}^{\Lambda} \in \mathbb{R}^{G \times 1}$ is a $K$-sparse vector. The number of non-zero elements $K$ is equal to the number of the targets, which is usually much smaller than the number of the grids $G$. Besides, the indices of the non-zero elements correspond to the indices of the grids containing the targets. Recalling the compressive sensing theory described in Section IV-A the sparse vector $\mathbf{h}$ can be recovered by using $L(L \ll G)$ measurements. That is to say, we can count and locate the multiple targets based on the energy values of $L$ randomly selected girds in the surveillance region. Let $\mathbf{P}_{l}^{r}=\left[x_{l}^{r}, y_{l}^{r}\right]^{T}$ represent center position of the $l$ th $(l=1, \ldots, L)$ picked grid, and $\mathbf{y}^{\Lambda}=\left[\Lambda\left(\mathbf{P}_{1}^{r}\right), \ldots, \Lambda\left(\mathbf{P}_{L}^{r}\right)\right]^{T} \in \mathbb{R}^{L \times 1}$ denote the measurement vector composed of the energy values of $L$ grids, then we have

$$
\mathbf{y}^{\Lambda}=\boldsymbol{\Phi}^{\Lambda} \mathbf{x}^{\Lambda}=\underbrace{\Phi^{\Lambda} \mathbf{\Psi}^{\Lambda}}_{\mathbf{A}^{\Lambda}} \mathbf{h}^{\Lambda}
$$

where $\boldsymbol{\Phi}^{\Lambda} \in \mathbb{R}^{L \times G}$ is the measurement matrix consisting of 0 and 1 . More specifically, for the $l$ th row of $\Phi^{\Lambda}$, only the element whose index in $G$ is equal to the $l$ th selected grid is 1 , and the rest elements are 0 . By multiplying the matrices $\boldsymbol{\Phi}^{\Lambda}$ and $\boldsymbol{\Psi}^{\Lambda}$, the dictionary matrix $\mathbf{A}^{\Lambda}=\left[\mathbf{a}_{1}^{\Lambda}, \ldots, \mathbf{a}_{G}^{\Lambda}\right] \in \mathbb{R}^{L \times G}$ can be constructed, which is essentially a recombination of $L$ rows in the matrix $\Psi^{\Lambda}$. In general, the measurement vector is corrupted with the noise, and thus (34) should be rewritten as

$$
\mathbf{y}^{\Lambda}=\mathbf{A}^{\Lambda} \mathbf{h}^{\Lambda}+\mathbf{z}^{\Lambda}
$$


where $\mathbf{z}^{\Lambda} \in \mathbb{R}^{L \times 1}$ is the noise vector. To facilitate the analysis, each column in $\mathbf{A}^{\Lambda}$ is normalized, and thus the measurement model 35 is reformulated as

$$
\mathbf{y}^{\Lambda}=\mathbf{A}^{\Lambda N} \mathbf{h}^{\Lambda N}+\mathbf{z}^{\Lambda}
$$

where $\mathbf{A}^{\Lambda N}=\left[\mathbf{a}_{1}^{\Lambda N}, \ldots, \mathbf{a}_{G}^{\Lambda N}\right]$, and the $g$ th $(g=1, \ldots, G)$ column $\mathbf{a}_{g}^{\Lambda N}=\mathbf{a}_{g}^{\Lambda} /\left\|\mathbf{a}_{g}^{\Lambda}\right\|$, which implies that $\left\|\mathbf{a}_{g}^{\Lambda N}\right\|=1$. Simultaneously, the non-zero elements in $\mathbf{h}^{\Lambda N}$ have scale changes compared with those in $\mathbf{h}^{\Lambda}$. Based on the model (36), the new issue is to develop a valid sparse vector recovery algorithm to support the multi-target counting and localization, and this is the focus of the next subsection.

\section{Matching Pursuit With Similarity Evaluation for Multi- Target Counting and Localization}

As stated in Section IV-A many sparse signal reconstruction methods have been proposed so far. Considering the specific scenario and the tradeoff between the computational complexity and the performance, a novel iterative greedy pursuit algorithm is designed to recover the sparse vector $\mathbf{h}^{\Lambda N}$ in 36 .

The backbone of the proposal is the classical MP algorithm. Let $\hat{\mathbf{h}}^{\Lambda N}=\mathbf{0}_{G \times 1}$ be the initialization of the reconstructed vector. For the $i$ th iteration, we first compute the vector $\boldsymbol{\mu}_{i}=$ $\left[\mathbf{a}_{1}^{\Lambda N}, \ldots, \mathbf{a}_{G}^{\Lambda N}\right]^{T} \mathbf{y}_{i-1}^{r e s} \in \mathbb{R}^{G \times 1}$, where $\mathbf{y}_{i-1}^{r e s}$ denotes the remaining part of the measurement vector $\mathbf{y}^{\Lambda}$ in the previous iteration, and it is initialized as $\mathbf{y}_{0}^{\text {res }}=\mathbf{y}^{\Lambda}$. Then we identify the maximum value in $\boldsymbol{\mu}_{i}$ as well as the corresponding index, which are represented as $\mu_{i}^{\max }$ and $g_{i}^{\max }$, respectively. Finally, the $g_{i}^{\max }$ th element of the reconstructed vector is updated as $\hat{\mathbf{h}}^{\Lambda N}\left(g_{i}^{\max }\right) \leftarrow \hat{\mathbf{h}}^{\Lambda N}\left(g_{i}^{\max }\right)+\mu_{i}^{\max }$, and the residual in the current iteration is updated as $\mathbf{y}_{i}^{r e s} \leftarrow \mathbf{y}_{i-1}^{r e s}-\mu_{i}^{\max } \mathbf{a}_{g_{i}^{\max }}^{\Lambda N}$. In essence, the procedure stated above is to find one column from the dictionary matrix $\mathbf{A}^{\Lambda N}$, which has the strongest positive correlation with the residual of $\mathbf{y}$. The gird whose index is equal to that of the selected column is temporarily considered containing a target. It is worth noting that the conventional MP algorithm finds the maximum value of the vector $\left|\boldsymbol{\mu}_{i}\right|$, while we only consider the non-negative elements. This is because the energy values of the grids are non-negative, which means that any element in the reconstructed vector $\hat{\mathbf{h}}^{\Lambda N}$ should not be less than zero.

Furthermore, in each iteration, we can also reconstruct the measurement vector as $\hat{\mathbf{y}}^{\Lambda}=\mathbf{A}^{\Lambda N} \hat{\mathbf{h}}^{\Lambda N}$. It is considered that if one gird that contains the target is picked correctly in the $i$ th iteration, then the reconstruction accuracy of $\hat{\mathbf{y}}^{\Lambda}$ should be improved significantly compared with the previous iteration; otherwise, the difference of the reconstruction accuracies between the two iterations is tiny. Based on this feature, we introduce the similarity evaluation strategy in the recovery process, which is used to judge whether the selected grid includes a real target. In particular, for the $i$ th iteration, the Pearson correlation coefficient is computed to quantify the similarity between $\mathbf{y}^{\Lambda}$ and $\hat{\mathbf{y}}^{\Lambda}$, which is expressed as

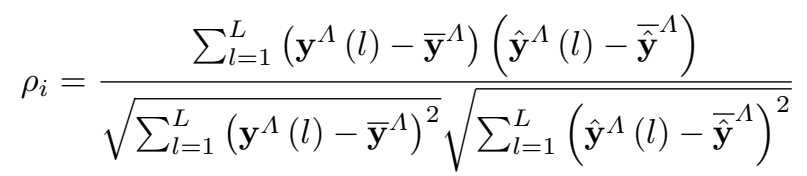

where $\overline{\mathbf{y}}^{\Lambda}$ and $\overline{\hat{\mathbf{y}}}^{\Lambda}$ stand for the means of $\mathbf{y}^{\Lambda}$ and $\hat{\mathbf{y}}^{\Lambda}$, respectively. The range of values for the similarity is $\rho_{i} \in[-1,1]$, and a larger value of $\rho_{i}$ indicates a higher reconstruction accuracy, and vice versa. Let $\Delta \rho=\rho_{i}-\rho_{i-1}$ be the similarity difference between the $i$ th and the $i-1$ th iterations, and then the target is confirmed to exist in the picked gird $\mathbf{P}_{g_{i}^{\text {max }}}$ when $\Delta \rho>\rho_{t h}$, where $\rho_{t h}$ is a predefined threshold. On the contrary, there is no target in the grid $\mathbf{P}_{g_{i}^{\max }}$ if $\Delta \rho \leq \rho_{t h}$ and $\mathbf{P}_{g_{i}^{\max }}$ has not been picked in the previous iterations. In addition, the value of $\hat{\mathbf{h}}^{\Lambda N}\left(g_{i}^{\max }\right)$ is set to 0 accordingly.

The iterative process exists when the algorithm meet one of the following conditions. 1) The number of non-zero elements in the reconstructed vector $\hat{\mathbf{h}}^{\Lambda N}$ is larger than the preset maximum number of the targets $\left.K_{\max } ; 2\right)$ the number of iterations exceeds the maximum number $i t e r_{\max }$; 3) the value of $\left\|\mathbf{y}_{i}^{r e s}\right\|$ is less than the product of the preset tolerance $y_{t o l}^{r e s}$ and $\left\|\mathbf{y}^{\Lambda}\right\|$. Finally, we acquire the reconstructed vector $\hat{\mathbf{h}}^{\Lambda N}$. It encodes the estimated number of the targets as well as the estimated target locations, which are denoted as $\hat{K}$ and $\hat{\mathbf{u}}=\left[\hat{\mathbf{u}}_{1}^{T}, \ldots, \hat{\mathbf{u}}_{\hat{K}}^{T}\right]^{T}$, respectively. For ease of interpretation, we present the pseudo code of the matching pursuit with similarity evaluation (abbreviated as MPWSE) in Algorithm 1.

\section{Simulation Results}

In this section, we assess the effectiveness of the proposed method via simulations. We first declare the scenario and signal settings, and then introduce several compared methods as well as evaluation indicators. Finally, a series of experiments are performed to examine the performance on multi-target counting and localization.

\section{A. Simulation Setup}

Without loss of generality, we consider a distributed MIMO radar system consisting of $M=4$ transmitters and $N=6$ receivers. Their positions are randomly selected in a square area with the coordinate range of $x \in[5,13] \mathrm{km}$ and $y \in$ $[3,11] \mathrm{km}$, and they are fixed in our simulations. The specific coordinates of each antenna are given in Tabel I In addition,

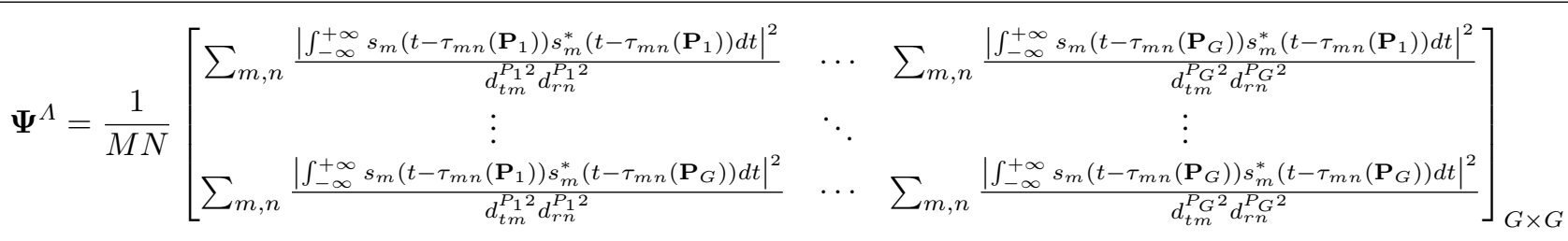




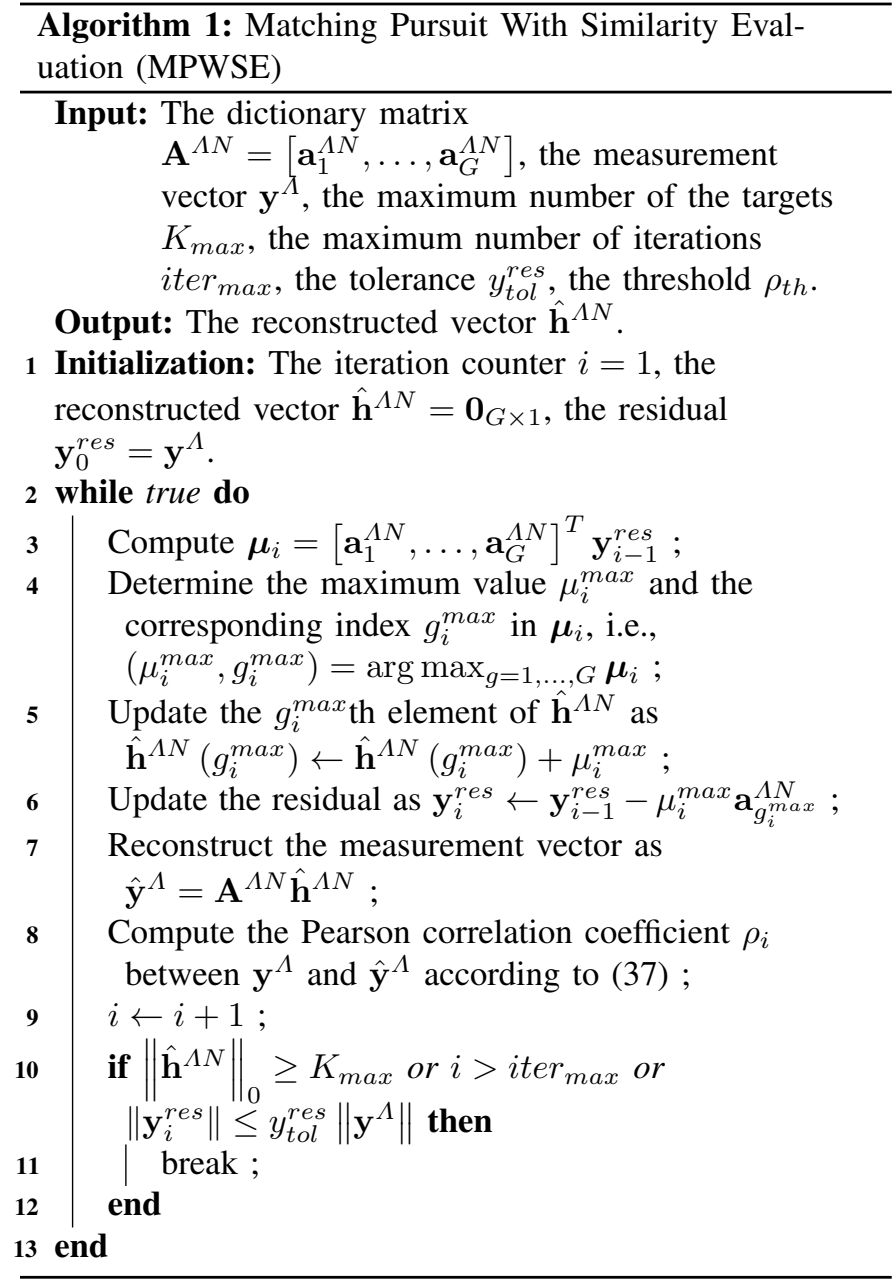

we suppose that the radius of the circular surveillance region $\Theta$ is $3 \mathrm{~km}$, and the center is $[0,0]^{T} \mathrm{~km}$. Unless otherwise specified, it is considered that there exist $K=3$ targets in the surveillance region $\Theta$, and their coordinates are set to $\mathbf{u}_{1}=[0.404,-1.208]^{T} \mathrm{~km}, \mathbf{u}_{2}=[-1.295,-0.412]^{T} \mathrm{~km}$, and $\mathbf{u}_{3}=[1.501,0.683]^{T} \mathrm{~km}$, respectively. We employ the Swerling I target model, and the amplitude of each target is modeled as a zero-mean complex Gaussian distribution. We further assume that the ratio of the amplitude variances is 1 : 1.2 : 0.5 . Considering the amplitude difference of different targets, we adopt the first target as the benchmark to define the SNR in the simulations, which is expressed as

$$
\begin{aligned}
& \mathrm{SNR}=\frac{E \sum_{m=1}^{M} \sum_{n=1}^{N} \sigma_{m n 1}^{2}}{M N \sigma_{\omega}^{2}} \\
& =\frac{E \sum_{m=1}^{M} \sum_{n=1}^{N} \sigma_{1}^{2} /\left(d_{t m 1}^{2} d_{r n 1}^{2}\right)}{M N \sigma_{\omega}^{2}} .
\end{aligned}
$$

For an intuitive explanation, the visualization of the discussed scenario is illustrated in Fig. 3 .

We consider that each transmitter emits linear frequency modulation (LFM) signal, which is commonly used in radar systems. The normalized lowpass equivalent form of the signal associated with the $m$ th transmitter is written as

$$
s_{m}(t)=\frac{1}{\sqrt{\mathcal{T}_{p}}} e^{j \pi \mathcal{B}\left(t^{2} / \mathcal{T}_{p}+\Delta f(m-1) t\right)}
$$

TABLE I

Positions of TransmitTERS AND RECEIVERS (IN KILOMETERS)

\begin{tabular}{ccccccc}
\hline & $\mathbf{T}_{1}$ & $\mathbf{T}_{2}$ & $\mathbf{T}_{3}$ & $\mathbf{T}_{4}$ & - & - \\
\hline$x_{m}^{t}$ & 5.610 & 11.239 & 8.507 & 10.788 & - & - \\
$y_{m}^{t}$ & 10.824 & 7.308 & 7.009 & 3.576 & - & - \\
\hline & $\mathbf{R}_{1}$ & $\mathbf{R}_{2}$ & $\mathbf{R}_{3}$ & $\mathbf{R}_{4}$ & $\mathbf{R}_{5}$ & $\mathbf{R}_{6}$ \\
\hline$x_{n}^{r}$ & 7.148 & 8.999 & 10.434 & 11.430 & 8.048 & 5.527 \\
$y_{n}^{r}$ & 5.305 & 10.277 & 4.707 & 6.617 & 10.450 & 3.199 \\
\hline
\end{tabular}

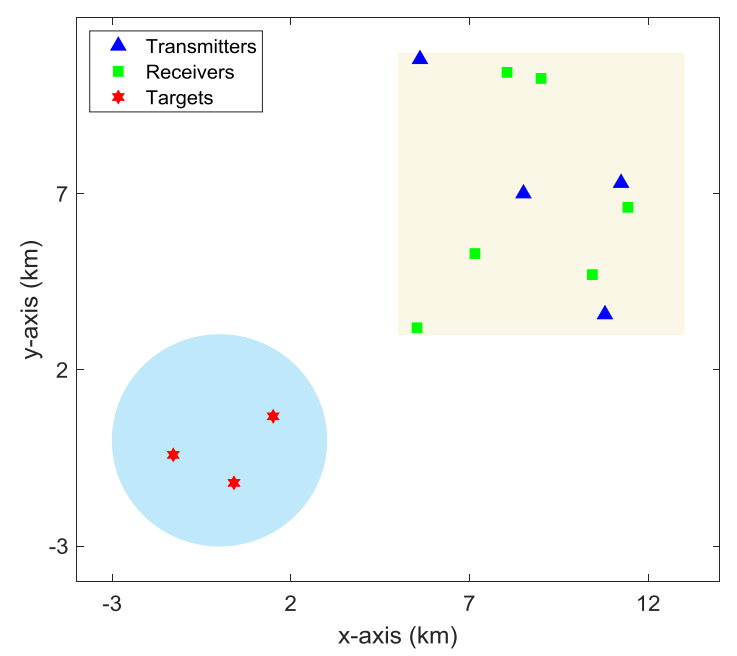

Fig. 3. Visualization of the discussed scenario.

where $\mathcal{T}_{p}$ and $\mathcal{B}$ denote the pulse width and the bandwidth, respectively. The term $\Delta f$ is the frequency interval parameter, which is used to keep the orthogonality of the transmitted signals. Here, these signals parameters are set as $\mathcal{T}_{p}=50 \mu \mathrm{s}$, $\mathcal{B}=1.6 \mathrm{MHz}$, and $\Delta f=1.2$. Moreover, the signal period $\mathcal{T}$ is set to $200 \mu$ s to ensure the valid matched filter outputs under the time delays caused by the targets, while the sampling rate $f_{s}$ is set to $7.36 \mathrm{MHz}$ (computed by $\mathcal{B}+(M-1) \Delta f \mathcal{B}$ ), so that the transmitted signals in discrete form can be approximately orthogonal. Based on the signal expression (39), we can obtain the analytical form of each element in the matrix $\Psi^{\Lambda}$, that is,

$$
\begin{aligned}
& \sum_{m=1}^{M} \sum_{n=1}^{N} \frac{\left|\int_{-\infty}^{+\infty} s_{m}\left(t-\tau_{m n}\left(\mathbf{P}_{g_{1}}\right)\right) s_{m}^{*}\left(t-\tau_{m n}\left(\mathbf{P}_{g_{2}}\right)\right) d t\right|^{2}}{d_{t m}^{P_{g_{1}} 2} d_{r n}^{P_{g_{1}} 2}} \\
& =\sum_{m=1}^{M} \sum_{n=1}^{N} \frac{\left|\left(1-\frac{\Delta \tau_{m n}}{\mathcal{T}_{p}}\right) \operatorname{sinc}\left(\frac{\mathcal{B}}{\mathcal{T}_{p}} \Delta \tau_{m n}\left(\mathcal{T}_{p}-\Delta \tau_{m n}\right)\right)\right|^{2}}{d_{t m}^{P_{g_{1}} 2} d_{r n}^{P_{g_{1}} 2}} \\
& g_{1}, g_{2}=1, \ldots, G
\end{aligned}
$$

where $\Delta \tau_{m n}=\left|\tau_{m n}\left(\mathbf{P}_{g_{2}}\right)-\tau_{m n}\left(\mathbf{P}_{g_{1}}\right)\right|$, and the details of the equation is given in Appendix B. By invoking (40), the construction of the dictionary matrix can be accelerated significantly in the simulations.

\section{B. Competitors and Performance Evaluation Criteria}

In our simulations, several state-of-the-art algorithms are introduced as the competitors for the purpose of performance 
comparison. To investigate the efficacy of the proposed energy modeling and compressive sensing framework for target localization, we employ two classical indirect localization algorithms as the compared methods, namely the LS method [16], and the TSWLS method [17], respectively. To examine the sparse vector recovery performance of the proposed MPWSE algorithm, we compare it with two well-known recovery algorithms, the CoSaMP [43] and the LASSO [46], respectively. It should be pointed out that the LS and the TSWLS methods cannot deal with the multi-target scenario, and thus we utilize $K$ single target signal models with the same noise level as that of the multi-target, so that the two indirect methods can locate the multiple targets sequentially. Moreover, considering that the number of non-zero elements (i.e., the sparsity level) needs to be known for the CoSaMP algorithm, we input the actual value of $K$ into it. That is to say, the multi-target counting performance of the CoSaMP is not evaluated. For the LASSO algorithm, we need to suppress the elements with tiny nonzero values before estimating the number and locations of the targets. Here, a simple approximation strategy is adopted [50]. Let $\hat{\mathbf{h}}^{\text {lasso }}$ represent the recovered sparse vector by the LASSO, then the suppressed vector $\hat{\mathbf{h}}^{\text {supp }}$ is computed as

$$
\hat{\mathbf{h}}^{\text {supp }}(g)= \begin{cases}0 & \text { if } \frac{\hat{\mathbf{h}}^{\text {lasso }}(g)}{\max \left(\left|\hat{\mathbf{h}}^{\text {lasso }}\right|\right)}<\lambda^{\text {lasso }} \\ \hat{\mathbf{h}}^{\text {lasso }}(g) & \text { otherwise }\end{cases}
$$

where $\lambda^{\text {lasso }}$ is a predefined threshold, which is set to 0.5 in our simulations. Owing to the non-negative property of the elements in the sparse vector, we also modify the CoSaMP by removing the absolute value operator in each iteration, and implement the LASSO with the non-negative constraint.

In order to evaluate the target localization performance quantitatively, we use the indicators of the root mean square error (RMSE) and the empirical cumulative density function (CDF). For the $k$ th target, their definitions are given as

$$
\begin{aligned}
& \operatorname{RMSE}\left(\mathbf{u}_{k}\right)=\sqrt{\frac{1}{N_{\text {ext }}} \sum_{i=1}^{N_{\text {ext }}}\left\|\hat{\mathbf{u}}_{k}^{(i)}-\mathbf{u}_{k}\right\|^{2}} \\
& \operatorname{CDF}\left(\mathbf{u}_{k}, e r r\right)=p\left(\left\|\hat{\mathbf{u}}_{k}^{(i)}-\mathbf{u}_{k}\right\| \leq e r r\right)
\end{aligned}
$$

where $N_{\text {ext }}$ is the number of times that the target $\mathbf{u}_{k}$ is picked in all the Monte Carlo runs, $\hat{\mathbf{u}}_{k}^{(i)}$ represents the estimation result of $\mathbf{u}_{k}$ in the $i$ th pick, and $e r r$ denotes the given estimation error. Here, it is necessary to clarify the adopted target association mechanism. We first compute all the pairwise distances between the estimated target locations and the actual target locations. Then we find the minimum value from the distances, and complete the matching between the two corresponding targets. Moreover, the remaining distances related to the two targets are eliminated. This procedure is repeated until all the estimated targets or the actual targets are matched.

Furthermore, the counting error (COE) is employed to assess the target counting performance, which is defined as

$$
\mathrm{COE}=\frac{\sum_{t=1}^{N_{M C}}\left|\hat{K}^{(t)}-K\right|}{N_{M C} \times K}
$$

TABLE II

PARAMETER SetTINGS OF MPWSE ALgORITHM

\begin{tabular}{cc}
\hline Maximum number of the targets $K_{\max }$ & 20 \\
Maximum number of the iterations iter $_{\max }$ & 20 \\
Tolerance $y_{\text {tol }}^{\text {res }}$ & $10^{-5}$ \\
Threshold $\rho_{t h}$ & 0.03 \\
\hline
\end{tabular}

TABLE III

COE VERSUS SNR FOR LASSO AND MPWSE

\begin{tabular}{ccc}
\hline \multirow{2}{*}{ SNR $(\mathrm{dB})$} & \multicolumn{2}{c}{ COE } \\
\cline { 2 - 3 } & LASSO & MPWSE \\
\hline-5 & 4.115 & 0.317 \\
0 & 3.230 & 0.355 \\
5 & 0.847 & 0.452 \\
10 & 0.419 & 0.158 \\
15 & 0.405 & 0.025 \\
20 & 0.382 & 0.013 \\
25 & 0.362 & 0.015 \\
30 & 0.367 & 0.017 \\
\hline
\end{tabular}

where $N_{M C}$ is the number of the Monte Carlo runs, and $\hat{K}^{(t)}$ represents the estimated number of the targets in the $t$ th run. Evidently, a small COE value reveals a high multi-target counting accuracy. Note that in our simulations, if there is no specific statement, the statistical result is acquired based on $N_{M C}=500$ trials.

\section{Multi-Target Counting and Localization Performance}

We first compare the localization performance of different methods with the variation of the SNR. For the compressive sensing recovery algorithms, we set the grid size to $50 \times 50 \mathrm{~m}^{2}$, which implies that the surveillance region $\Theta$ is divided into $G=11287$ grids. Besides, we randomly select $L=\lfloor 30 K \log (G / K)\rceil=741$ grids from $G$ to construct the dictionary matrix. The parameter settings of the MPWSE are listed in Table [I] and they are consistent with the subsequent simulations. Fig. 4 shows the results of RMSE versus SNR for the three targets. It can be seen that the compressive sensing recovery algorithms outperform the indirect localization algorithms significantly. Since the time delay with regard to the single transmitter-receiver path is hard to be estimated accurately in terms of the tested SNR range, while this information is unnecessary for the compressive sensing recovery methods. It is also noticed that the LASSO yields the best performance when SNR $\leq 5 \mathrm{~dB}$, but the MPWSE has more superior performance with the rise of the SNR, and its localization accuracies for all the three targets are close to the corresponding theoretical lower bounds when SNR $\geq 15 \mathrm{~dB}$. In order to demonstrate the multi-target counting performance, we give the COE values under different SNRs in Table III Considering the lack of the multi-target counting ability of the indirect methods and the CoSaMP, Table III only contains the results of the LASSO and the MPWSE. We can observe that the MPWSE has significantly higher counting accuracy than the LASSO for each SNR. In particular, the COE values of the MPWSE are less than 0.02 when $S N R \geq 20 \mathrm{~dB}$, which reflects its remarkable counting performance. 


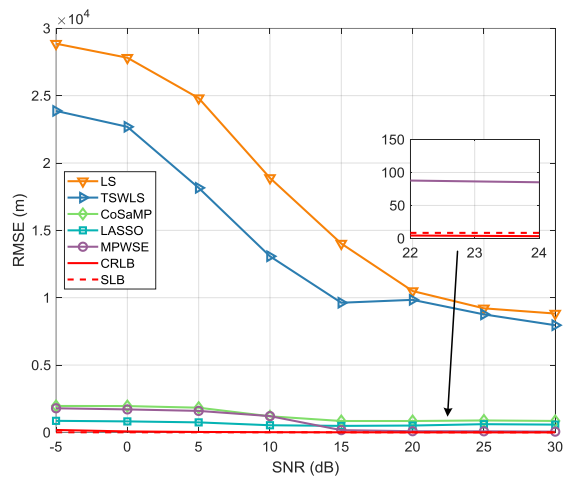

(a)

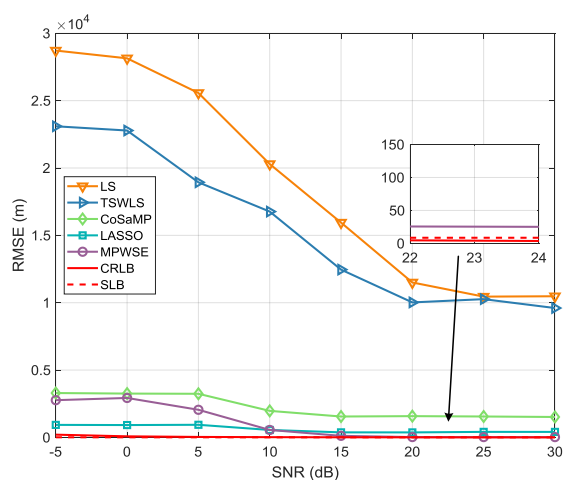

(b)

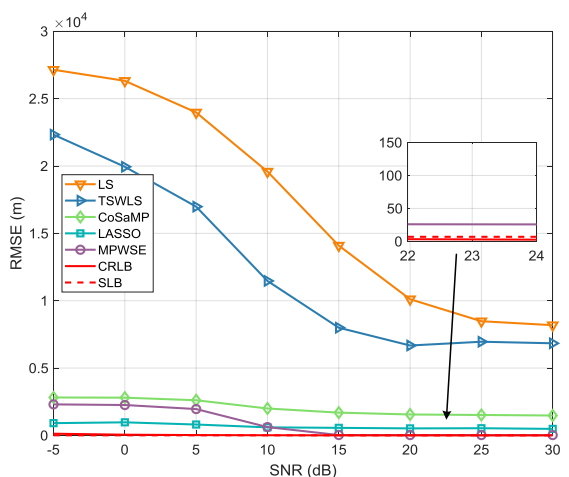

(c)

Fig. 4. RMSE versus SNR for different methods. (a) The 1st target. (b) The 2nd target. (c) The 3rd target.

TABLE IV

Average RunNing TIMES (SECONDS) FOR COSAMP, LASSO, AND MPWSE

\begin{tabular}{cccc}
\hline \multirow{2}{*}{ SNR $(\mathrm{dB})$} & \multicolumn{3}{c}{ Running Time } \\
\cline { 2 - 4 } & CoSaMP & LASSO & MPWSE \\
\hline-5 & 0.206 & 7.232 & 0.073 \\
0 & 0.207 & 7.021 & 0.075 \\
5 & 0.202 & 5.956 & 0.072 \\
10 & 0.202 & 3.979 & 0.074 \\
15 & 0.203 & 2.064 & 0.074 \\
20 & 0.205 & 1.041 & 0.076 \\
25 & 0.208 & 0.597 & 0.073 \\
30 & 0.204 & 0.420 & 0.072 \\
\hline
\end{tabular}

Then, we perform the experiment with random multi-target distribution. Here, we fix the SNR to $20 \mathrm{~dB}$, and the other parameter settings are the same as the previous experiment. However, in each independent trial, the actual positions of the three targets are given randomly within the surveillance region $\Theta$. Fig. 5 illustrates the CDF results of different methods for the three targets. In general, we see that the proposed MPWSE still produces good performance compared with the other methods.

In the end, the computational complexities of the compressive sensing recovery methods are evaluated by comparing their time consumptions. We adopt the same settings as the second experiment. For each recovery algorithm, we compute the average running times over a total of 30 trials under different SNRs. The statistics are given in Table IV] It is seen that the LASSO requires the most computational cost in the three algorithms, especially for the case of low SNR. In contrast, the MPWSE is faster than the other two algorithms, and this is also a major advantage of it.

\section{Robustness Analysis}

In this subsection, we study the performance of the MPWSE algorithm under different structures of the dictionary matrix or the sparse vector, that is, analyzing the robustness of the algorithm. The simulations are conducted from three aspects, which are described separately.
1) Effect of grid size: The influence of the grid size $\Delta d \times \Delta d$ on the multi-target localization performance is discussed in this experiment. We fix the number of measurements to $L=800$, and change the grid size from $30 \times 30 \mathrm{~m}^{2}$ to $100 \times 100 \mathrm{~m}^{2}$ at the step size of $10 \times 10 \mathrm{~m}^{2}$. This results in the total number of girds $G$ varying from 31415 to 2819 . Fig. 6 depicts the performance of RMSE for the three targets when SNR $=10 \mathrm{~dB}$ and $20 \mathrm{~dB}$. It is observed that the RMSEs of different targets are stable within the tested grid size range, which means that the localization accuracy of the proposal cannot be improved effectively by merely shrinking the grid size. For the case of SNR $=10 \mathrm{~dB}$, there exist obvious performance differences among the three targets, especially the result of the third target is better than the other two targets remarkably. In contrast, their localization accuracies are relatively close when $\mathrm{SNR}=20 \mathrm{~dB}$, and each target acquires a considerable performance improvement with the increase of the SNR.

2) Effect of number of measurements: Next, we perform the experiment to investigate the dependence between the localization performance and the number of the measurements $L$. Herein, the grid size is set as $50 \times 50 \mathrm{~m}^{2}$, while the number of measurements increases from $\lfloor 10 K \log (G / K)\rceil=247$ to $\lfloor 50 K \log (G / K)\rceil=1235$ at the step size of $5 K \log (G / K) \approx$ 123. Similar to the previous experiment, we present the RMSE results for the three targets with SNR $=10 \mathrm{~dB}$ and $20 \mathrm{~dB}$ in Fig. 7. It can be noticed that with the increase of the number of the measurements, the localization accuracy improvement of each target is limited. Since all the tested values satisfy the requirement of $L=\mathcal{O}(K \log (G / K))$, we consider that the experimental results conform to the compressive sensing theory. We can also see some features consistent with the previous experiment from Fig. 7. For example, the increase of the SNR leads to a significant boost on the localization accuracy for each target. In addition, when SNR $=20 \mathrm{~dB}$, the performance gaps among different targets are smaller than that of SNR $=10 \mathrm{~dB}$

3) Effect of number of targets: Finally, the impact of the number of the targets $K$ on the multi-target counting performance is discussed. In the experiment, we set the grid size $\Delta d \times \Delta d$ and the number of measurements $L$ as $50 \times 50 \mathrm{~m}^{2}$ and $\lfloor 30 K \log (G / K)\rceil$, respectively. We consider some scenarios 


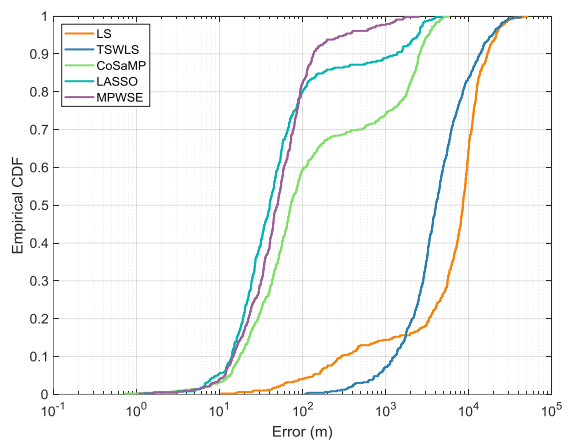

(a)

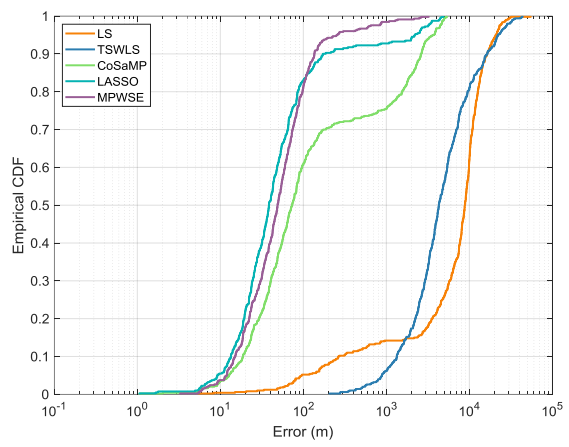

(b)

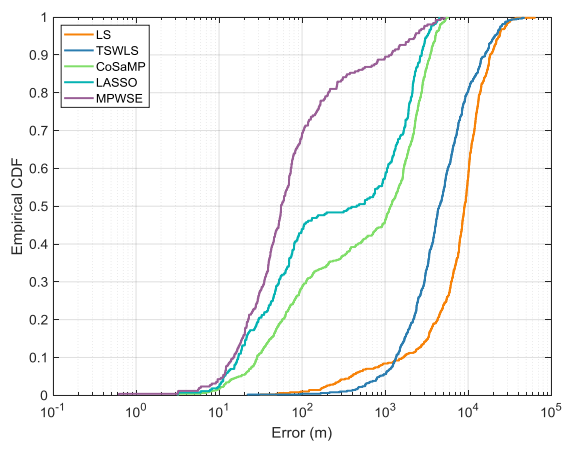

(c)

Fig. 5. Empirical $\mathrm{CDF}$ of different methods under $\mathrm{SNR}=20 \mathrm{~dB}$. (a) The 1st target. (b) The 2nd target. (c) The 3rd target.

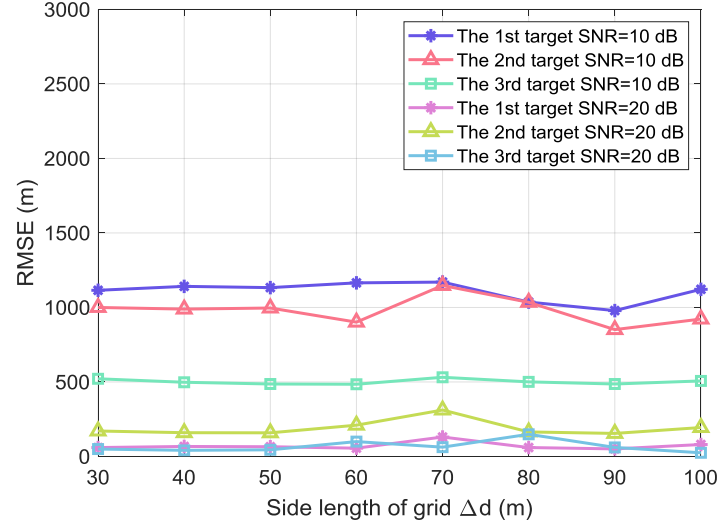

Fig. 6. RMSE versus grid size $\Delta d \times \Delta d$ for the three targets when $\mathrm{SNR}=10$ $\mathrm{dB}$ and $20 \mathrm{~dB}$.

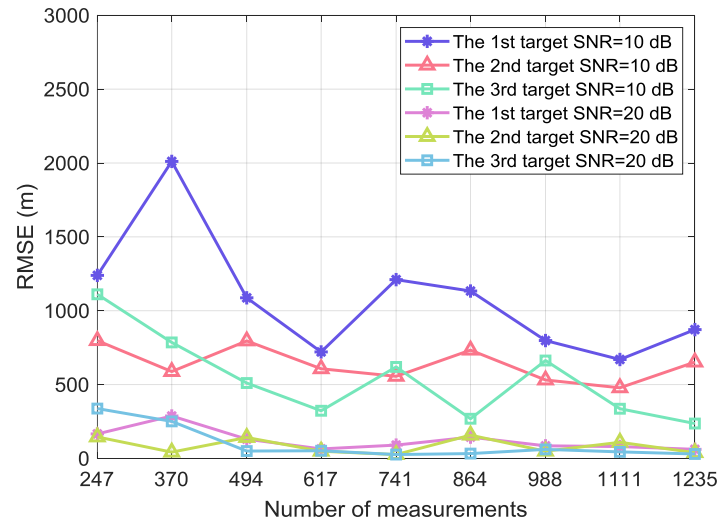

Fig. 7. RMSE versus number of measurements for the three targets when $\mathrm{SNR}=10 \mathrm{~dB}$ and $20 \mathrm{~dB}$.

with different numbers of the targets, which increase from 3 to 10. For each case, all the targets have the same amplitude variance, and their positions are randomly produced in each Monte Carlo trial. Table $\mathrm{V}$ presents the COE results when SNR $=10 \mathrm{~dB}$ and $20 \mathrm{~dB}$. On the whole, we can observe that the COE values are relatively higher when the number of the targets is lager. This indicates that accurate counting is very challenging for the case of large number of targets. It is also seen from Table $\mathrm{V}$ that the counting performance is close for the two tested SNRs. Moreover, we can further
TABLE V

COE VERSUS NUMBER OF TARGETS

\begin{tabular}{ccc}
\hline \multirow{2}{*}{ Number of targets $K$} & \multicolumn{2}{c}{ COE } \\
\cline { 2 - 3 } & SNR $=10 \mathrm{~dB}$ & $\mathrm{SNR}=20 \mathrm{~dB}$ \\
\hline 3 & 0.308 & 0.261 \\
4 & 0.166 & 0.186 \\
5 & 0.426 & 0.369 \\
6 & 0.379 & 0.382 \\
7 & 0.614 & 0.599 \\
8 & 0.566 & 0.572 \\
9 & 0.584 & 0.618 \\
10 & 0.576 & 0.578 \\
\hline
\end{tabular}

notice that when $K=3$, the statistic in Table $\mathrm{V}$ is inferior to that in Table III for the same SNR, which is caused by different target generation modes.

\section{CONCLUSION}

We focused on the multi-target counting and localization based on the distributed MIMO radar in this paper. First, in order to shed light on the localization problem, we utilized the tools of signal estimation theory to derive the theoretical lower bound of the multi-target localization. Next, we proposed a novel energy modeling and compressive sensing based framework, which transformed the issue of multi-target counting and localization into a sparse vector recovery problem. Compared with the ordinary direct localization methods, one noticeable advantage of the developed framework was the low requirement in terms of the data communication. On this foundation, we could acquire the estimations of the number of the targets and the target positions by using the designed MPWSE recovery algorithm. The effectiveness and robustness of the proposed method were verified by a series of experiments. The simulation results indicated that our proposal possessed outstanding counting and localization performance, as well as low computational cost. Moreover, it also emerged strong adaptability to different scenario conditions.

\section{ACKNOWLEDGMENTS}

We would like to thank David Donoho et al. for providing the source codes of the LASSO algorithm at http://sparselab.stanford.edu. 
APPENDiX A

DERIVATION OF (15)

According to the expression of $\boldsymbol{\Sigma}$ in $(10),(15)$ can be reformulated as

$$
[\mathbf{J}(\boldsymbol{\zeta})]_{q, q^{\prime}}=\sum_{n=1}^{N} \operatorname{tr}\left(\boldsymbol{\Sigma}_{n}^{-1} \frac{\partial \boldsymbol{\Sigma}_{n}}{\partial \zeta_{q}} \boldsymbol{\Sigma}_{n}^{-1} \frac{\partial \boldsymbol{\Sigma}_{n}}{\partial \zeta_{q^{\prime}}}\right)
$$

where $\boldsymbol{\Sigma}_{n}=E \mathbf{S}_{n} \boldsymbol{\Sigma}_{\alpha_{n}} \mathbf{S}_{n}^{H}+\boldsymbol{\Sigma}_{\omega_{n}}$, by applying the Woodbury identity [51], we have

$$
\begin{aligned}
& \boldsymbol{\Sigma}_{n}^{-1}=\left(E \mathbf{S}_{n} \boldsymbol{\Sigma}_{\alpha_{n}} \mathbf{S}_{n}^{H}+\boldsymbol{\Sigma}_{\omega_{n}}\right)^{-1} \\
& =\frac{1}{\sigma_{\omega}^{2}} \mathbf{I}_{I}-\frac{E}{\sigma_{\omega}^{4}} \mathbf{S}_{n}\left(\boldsymbol{\Sigma}_{\alpha_{n}}^{-1}+\frac{E}{\sigma_{\omega}^{2}} \mathbf{S}_{n}^{H} \mathbf{S}_{n}\right)^{-1} \mathbf{S}_{n}^{H} .
\end{aligned}
$$

In addition, notice that

$$
E \mathbf{S}_{n} \boldsymbol{\Sigma}_{\alpha_{n}} \mathbf{S}_{n}^{H}+\boldsymbol{\Sigma}_{\omega_{n}}=\sum_{m=1}^{M} \sum_{k=1}^{K} E \sigma_{m n k}^{2} \mathbf{s}_{m n k} \mathbf{s}_{m n k}^{H}+\sigma_{\omega}^{2} \mathbf{I}_{I}
$$

and thus its derivative with respect to a given element $\zeta_{q}=$ $\tau_{m n k}$ is

$$
\begin{aligned}
& \frac{\partial \boldsymbol{\Sigma}_{n}}{\partial \zeta_{q}}=\frac{\partial\left(E \mathbf{S}_{n} \boldsymbol{\Sigma}_{\alpha_{n}} \mathbf{S}_{n}^{H}+\boldsymbol{\Sigma}_{\omega_{n}}\right)}{\partial \tau_{m n k}} \\
& =E \sigma_{m n k}^{2}\left(\frac{\partial \mathbf{s}_{m n k}}{\partial \tau_{m n k}} \mathbf{s}_{m n k}^{H}+\mathbf{s}_{m n k} \frac{\partial \mathbf{s}_{m n k}^{H}}{\partial \tau_{m n k}}\right) .
\end{aligned}
$$

For a given sampling index in $\mathbf{s}_{m n k}$, the derivative with respect to $\tau_{m n k}$ can be written as

$$
\frac{\partial s_{m}\left[i-i_{m n k}\right]}{\partial \tau_{m n k}}=-\frac{1}{\sqrt{f_{s}}} \frac{\partial s_{m}(t)}{\partial t}
$$

and the analytical form of 490 is determined by the specific form of the signal $s_{m}(t)$.

Finally, we can obtain the expression of $\mathbf{J}(\boldsymbol{\zeta})$ as

$$
\mathbf{J}(\boldsymbol{\zeta})=\operatorname{blkdiag}\left(\mathbf{V}_{1}, \ldots, \mathbf{V}_{N}\right)
$$

where $\mathbf{V}_{n} \in \mathbb{C}^{M K \times M K}$ is the block matrix associated with the $n$th receiver, and the element in $\mathbf{V}_{n}$ can be expressed as

$$
\begin{aligned}
& {\left[\mathbf{V}_{n}\right]_{q_{V}, q_{V}^{\prime}}=E^{2} \sigma_{m n k}^{2} \sigma_{m^{\prime} n k^{\prime}}^{2} \operatorname{tr}(} \\
& \times\left(\frac{1}{\sigma_{\omega}^{2}} \mathbf{I}_{I}-\frac{E}{\sigma_{\omega}^{4}} \mathbf{S}_{n}\left(\boldsymbol{\Sigma}_{\alpha_{n}}^{-1}+\frac{E}{\sigma_{\omega}^{2}} \mathbf{S}_{n}^{H} \mathbf{S}_{n}\right)^{-1} \mathbf{S}_{n}^{H}\right) \\
& \times\left(\frac{\partial \mathbf{s}_{m n k}}{\partial \tau_{m n k}} \mathbf{s}_{m n k}^{H}+\mathbf{s}_{m n k} \frac{\partial \mathbf{s}_{m n k}^{H}}{\partial \tau_{m n k}}\right) \\
& \times\left(\frac{1}{\sigma_{\omega}^{2}} \mathbf{I}_{I}-\frac{E}{\sigma_{\omega}^{4}} \mathbf{S}_{n}\left(\boldsymbol{\Sigma}_{\alpha_{n}}^{-1}+\frac{E}{\sigma_{\omega}^{2}} \mathbf{S}_{n}^{H} \mathbf{S}_{n}\right)^{-1} \mathbf{S}_{n}^{H}\right) \\
& \left.\times\left(\frac{\partial \mathbf{s}_{m^{\prime} n k^{\prime}}}{\partial \tau_{m^{\prime} n k^{\prime}}} \mathbf{s}_{m^{\prime} n k^{\prime}}^{H}+\mathbf{s}_{m^{\prime} n k^{\prime}} \frac{\partial \mathbf{s}_{m^{\prime} n k^{\prime}}^{H}}{\partial \tau_{m^{\prime} n k^{\prime}}}\right)\right)
\end{aligned}
$$

where $q_{V}=(m-1) \times K+k$ and $q_{V}^{\prime}=\left(m^{\prime}-1\right) \times K+k^{\prime}$.

\section{APPENDIX B}

COMPUTATION OF (40)

To simplify the notations, we first define $\kappa=\mathcal{B} / \mathcal{T}_{p}$, and $\Delta F_{m}=(m-1) \Delta f \mathcal{B}$, then the LFM signal (39) can be expressed as

$$
s_{m}(t)=\frac{1}{\sqrt{\mathcal{T}_{p}}} e^{j \pi\left(\kappa t^{2}+\Delta F_{m} t\right)}
$$

Substituting (52) into the first term of $(40)$, we have

$$
\begin{aligned}
& \sum_{m=1}^{M} \sum_{n=1}^{N} \frac{\left|\int_{-\infty}^{+\infty} s_{m}\left(t-\tau_{m n}\left(\mathbf{P}_{g_{1}}\right)\right) s_{m}^{*}\left(t-\tau_{m n}\left(\mathbf{P}_{g_{2}}\right)\right) d t\right|^{2}}{d_{t m}^{P_{g_{1}} 2} d_{r n}^{P_{g_{1}} 2}} \\
& =\sum_{m=1}^{M} \sum_{n=1}^{N} \frac{1}{d_{t m}^{P_{g_{1}} 2} d_{r n}^{P_{g_{1}} 2}} \mid \frac{1}{\mathcal{T}_{p}} \int_{-\infty}^{+\infty} \\
& e^{j \pi\left(\kappa\left(t-\tau_{m n}\left(\mathbf{P}_{g_{1}}\right)\right)^{2}+\Delta F_{m}\left(t-\tau_{m n}\left(\mathbf{P}_{g_{1}}\right)\right)\right)} \\
& \times\left. e^{-j \pi\left(\kappa\left(t-\tau_{m n}\left(\mathbf{P}_{g_{1}}\right)\right)^{2}+\Delta F_{m}\left(t-\tau_{m n}\left(\mathbf{P}_{g_{1}}\right)\right)\right)} d t\right|^{2} \\
& =\sum_{m=1}^{M} \sum_{n=1}^{N} \frac{1}{d_{t m}^{P_{g_{1}} 2} d_{r n}^{P_{g_{1}} 2}} \mid \frac{1}{\mathcal{T}_{p}} \\
& e^{j \pi\left(\tau_{m n}\left(\mathbf{P}_{g_{1}}\right)-\tau_{m n}\left(\mathbf{P}_{g_{2}}\right)\right)\left(\kappa\left(\tau_{m n}\left(\mathbf{P}_{g_{1}}\right)+\tau_{m n}\left(\mathbf{P}_{g_{2}}\right)\right)-\Delta F_{m}\right)} \\
& \times \int_{\max \left(\tau_{m n}\left(\mathbf{P}_{g_{1}}\right), \tau_{m n}\left(\mathbf{P}_{g_{2}}\right)\right)}^{\min \left(\tau_{m n}\left(\mathbf{P}_{g_{1}}\right), \tau_{m n}\left(\mathbf{P}_{g_{2}}\right)\right)+\mathcal{T}_{p}} \\
& \left.e^{j 2 \pi \kappa\left(\tau_{m n}\left(\mathbf{P}_{g_{2}}\right)-\tau_{m n}\left(\mathbf{P}_{g_{1}}\right)\right) t} d t\right|^{2} \\
& =\sum_{m=1}^{M} \sum_{n=1}^{N} \frac{1}{d_{t m}^{P_{g_{1}} 2} d_{r n}^{P_{g_{1}} 2}}\left|\frac{1}{\mathcal{T}_{p}} \int_{\Delta \tau_{m n}}^{\mathcal{T}_{p}} e^{j 2 \pi \kappa \Delta \tau_{m n} t} d t\right|^{2} \\
& =\sum_{m=1}^{M} \sum_{n=1}^{N} \frac{1}{d_{t m}^{P_{g_{1}} 2} d_{r n}^{P_{g_{1}} 2}}\left|\frac{1}{\mathcal{T}_{p}} \times \frac{e^{j 2 \pi \kappa \Delta \tau_{m n} \mathcal{T}_{p}}-e^{j 2 \pi \kappa \Delta \tau_{m n}^{2}}}{j 2 \pi \kappa \Delta \tau_{m n}}\right|^{2} \\
& =\sum_{m=1}^{M} \sum_{n=1}^{N} \frac{1}{d_{t m}^{P_{g_{1}} 2} d_{r n}^{P_{g_{1}} 2}} \mid\left(1-\frac{\Delta \tau_{m n}}{\mathcal{T}_{p}}\right) \\
& \times\left.\frac{e^{j \pi \kappa \Delta \tau_{m n}\left(\mathcal{T}_{p}-\Delta \tau_{m n}\right)}-e^{-j \pi \kappa \Delta \tau_{m n}\left(\mathcal{T}_{p}-\Delta \tau_{m n}\right)}}{j 2 \pi \kappa \Delta \tau_{m n}\left(\mathcal{T}_{p}-\Delta \tau_{m n}\right)}\right|^{2} \\
& =\sum_{m=1}^{M} \sum_{n=1}^{N} \frac{\left|\left(1-\frac{\Delta \tau_{m n}}{\mathcal{T}_{p}}\right) \operatorname{sinc}\left(\frac{\mathcal{B}}{\mathcal{T}_{p}} \Delta \tau_{m n}\left(\mathcal{T}_{p}-\Delta \tau_{m n}\right)\right)\right|^{2}}{d_{t m}^{P_{g_{1}} 2} d_{r n}^{P_{g_{1}} 2}} \\
& g_{1}, g_{2}=1, \ldots, G \text {. }
\end{aligned}
$$

Thus, the expression (40) holds.

\section{REFERENCES}

[1] E. Fishler, A. Haimovich, R. S. Blum, L. J. Cimini, D. Chizhik, and R. A. Valenzuela, "Spatial diversity in radars-models and detection performance," IEEE Transactions on Signal Processing, vol. 54, no. 3, pp. 823-838, 2006.

[2] J. Li and P. Stoica, "MIMO radar with colocated antennas," IEEE Signal Processing Magazine, vol. 24, no. 5, pp. 106-114, 2007.

[3] A. M. Haimovich, R. S. Blum, and L. J. Cimini, "MIMO radar with widely separated antennas," IEEE Signal Processing Magazine, vol. 25, no. 1, pp. 116-129, 2007.

[4] A. De Maio and M. Lops, "Design principles of MIMO radar detectors," IEEE Transactions on Aerospace and Electronic Systems, vol. 43, no. 3, pp. 886-898, 2007. 
[5] H. Godrich, A. M. Haimovich, and R. S. Blum, "Target localization accuracy gain in MIMO radar-based systems," IEEE Transactions on Information Theory, vol. 56, no. 6, pp. 2783-2803, 2010.

[6] Q. He, R. S. Blum, and A. M. Haimovich, "Noncoherent MIMO radar for location and velocity estimation: More antennas means better performance," IEEE Transactions on Signal Processing, vol. 58, no. 7, pp. 3661-3680, 2010.

[7] R. Niu, R. S. Blum, P. K. Varshney, and A. L. Drozd, "Target localization and tracking in noncoherent multiple-input multiple-output radar systems," IEEE Transactions on Aerospace and Electronic Systems, vol. 48, no. 2, pp. 1466-1489, 2012.

[8] S. G. Dontamsetti and R. V. R. Kumar, "A distributed MIMO radar with joint optimal transmit and receive signal combining," IEEE Transactions on Aerospace and Electronic Systems, vol. 57, no. 1, pp. 623-635, 2020.

[9] P. Wang and H. Li, "Target detection with imperfect waveform separation in distributed MIMO radar," IEEE Transactions on Signal Processing, vol. 68 , pp. 793-807, 2020.

[10] H. Li, F. Wang, C. Zeng, and M. Govoni, "Signal detection in distributed MIMO radar with non-orthogonal waveforms and sync errors," IEEE Transactions on Signal Processing, 2021.

[11] Q. He, N. H. Lehmann, R. S. Blum, and A. M. Haimovich, "MIMO radar moving target detection in homogeneous clutter," IEEE Transactions on Aerospace and Electronic Systems, vol. 46, no. 3, pp. 1290-1301, 2010.

[12] C. Y. Chong, F. Pascal, J.-P. Ovarlez, and M. Lesturgie, "MIMO radar detection in non-Gaussian and heterogeneous clutter," IEEE Journal of Selected Topics in Signal Processing, vol. 4, no. 1, pp. 115-126, 2010.

[13] P. Wang, H. Li, and B. Himed, "Moving target detection using distributed MIMO radar in clutter with nonhomogeneous power," IEEE Transactions on Signal Processing, vol. 59, no. 10, pp. 4809-4820, 2011.

[14] N. Li, H. Yang, G. Cui, L. Kong, and Q. H. Liu, "Adaptive twostep Bayesian MIMO detectors in compound-Gaussian clutter," Signal Processing, vol. 161, pp. 1-13, 2019.

[15] L. Zhu, G. Wen, Y. Liang, D. Luo, and H. Song, "Parametric Wald test for target detection with distributed MIMO radar in partially mixing homogeneous and non-homogeneous environments," IET Radar, Sonar \& Navigation, 2021.

[16] M. Dianat, M. R. Taban, J. Dianat, and V. Sedighi, "Target localization using least squares estimation for MIMO radars with widely separated antennas," IEEE Transactions on Aerospace and Electronic Systems, vol. 49, no. 4, pp. 2730-2741, 2013.

[17] Y. Du and P. Wei, "An explicit solution for target localization in noncoherent distributed MIMO radar systems," IEEE Signal Processing Letters, vol. 21, no. 9, pp. 1093-1097, 2014.

[18] H. Song, G. Wen, Y. Liang, L. Zhu, and D. Luo, "Target localization and clock refinement in distributed MIMO radar systems with time synchronization errors," IEEE Transactions on Signal Processing, vol. 69, pp. 3088-3103, 2021.

[19] M. Sadeghi, F. Behnia, R. Amiri, and A. Farina, "Target localization geometry gain in distributed MIMO radar," IEEE Transactions on Signal Processing, vol. 69, pp. 1642-1652, 2021.

[20] Q. He, R. S. Blum, H. Godrich, and A. M. Haimovich, "Target velocity estimation and antenna placement for MIMO radar with widely separated antennas," IEEE Journal of Selected Topics in Signal Processing, vol. 4, no. 1, pp. 79-100, 2010.

[21] H. Godrich, A. P. Petropulu, and H. V. Poor, "Power allocation strategies for target localization in distributed multiple-radar architectures," IEEE Transactions on Signal Processing, vol. 59, no. 7, pp. 3226-3240, 2011.

[22] H. Yang and J. Chun, "An improved algebraic solution for moving target localization in noncoherent MIMO radar systems," IEEE Transactions on Signal Processing, vol. 64, no. 1, pp. 258-270, 2015.

[23] H. Song, G. Wen, L. Zhu, and D. Li, "A novel TSWLS method for moving target localization in distributed MIMO radar systems," IEEE Communications Letters, vol. 23, no. 12, pp. 2210-2214, 2019.

[24] C.-H. Park and J.-H. Chang, "Closed-form localization for distributed MIMO radar systems using time delay measurements," IEEE Transactions on Wireless Communications, vol. 15, no. 2, pp. 1480-1490, 2015

[25] J. Liang, C. S. Leung, and H. C. So, "Lagrange programming neural network approach for target localization in distributed MIMO radar," IEEE Transactions on Signal Processing, vol. 64, no. 6, pp. 1574-1585, 2015.

[26] J. Liang, D. Wang, L. Su, B. Chen, H. Chen, and H. C. So, "Robust MIMO radar target localization via nonconvex optimization," Signal Processing, vol. 122, pp. 33-38, 2016.

[27] L. Zhu, G. Wen, H. Song, Y. Liang, and D. Luo, "Robust moving target localization in distributed MIMO radars via iterative lagrange programming neural network," IEEE Sensors Journal, vol. 20, no. 21, pp. $13007-13017,2020$.
[28] R. Amiri, F. Behnia, and A. Noroozi, "Efficient joint moving target and antenna localization in distributed MIMO radars," IEEE Transactions on Wireless Communications, vol. 18, no. 9, pp. 4425-4435, 2019.

[29] S. Gogineni and A. Nehorai, "Target estimation using sparse modeling for distributed MIMO radar," IEEE Transactions on Signal Processing, vol. 59, no. 11, pp. 5315-5325, 2011.

[30] W. Yi, T. Zhou, Y. Ai, and R. S. Blum, "Suboptimal low complexity joint multi-target detection and localization for non-coherent MIMO radar with widely separated antennas," IEEE Transactions on Signal Processing, vol. 68, pp. 901-916, 2020.

[31] Z. Yu, J. Li, Q. Guo, and J. Ding, "Efficient direct target localization for distributed MIMO radar with expectation propagation and belief propagation," IEEE Transactions on Signal Processing, vol. 69, pp. 4055-4068, 2021.

[32] J. Liu, Y. Xue, K. Ren, J. Song, C. Windmill, and P. Merritt, "Highperformance time-series quantitative retrieval from satellite images on a gpu cluster," IEEE Journal of Selected Topics in Applied Earth Observations and Remote Sensing, vol. 12, no. 8, pp. 2810-2821, 2019.

[33] S. G. Mallat and Z. Zhang, "Matching pursuits with time-frequency dictionaries," IEEE Transactions on Signal Processing, vol. 41, no. 12, pp. 3397-3415, 1993.

[34] M. A. Richards, Fundamentals of radar signal processing. McGrawHill Education, 2014.

[35] S. M. Kay, Fundamentals of statistical signal processing: estimation theory. Prentice-Hall, Inc., 1993.

[36] H. Godrich, A. M. Haimovich, and R. S. Blum, "Cramer Rao bound on target localization estimation in MIMO radar systems," in 2008 42nd Annual Conference on Information Sciences and Systems. IEEE, 2008, pp. 134-139.

[37] C. Wei, Q. He, and R. S. Blum, "Cramer-rao bound for joint location and velocity estimation in multi-target non-coherent MIMO radars," in 2010 44th Annual Conference on Information Sciences and Systems (CISS). IEEE, 2010, pp. 1-6.

[38] L. C. Andrews, Special functions of mathematics for engineers. SPIE Press, 1998, vol. 49.

[39] B. Zhang, X. Cheng, N. Zhang, Y. Cui, Y. Li, and Q. Liang, "Sparse target counting and localization in sensor networks based on compressive sensing," in 2011 Proceedings IEEE INFOCOM. IEEE, 2011, pp. 22552263.

[40] V. Cevher, M. F. Duarte, and R. G. Baraniuk, "Distributed target localization via spatial sparsity," in 2008 16th European Signal Processing Conference. IEEE, 2008, pp. 1-5.

[41] J. A. Tropp and S. J. Wright," "Computational methods for sparse solution of linear inverse problems," Proceedings of the IEEE, vol. 98, no. 6, pp. 948-958, 2010.

[42] S. Wenze and W. Zhihui, "Advances and perspectives on compressed sensing theory," Journal of Image and Graphics, vol. 17, no. 1, pp. $1-12,2012$.

[43] D. Needell and J. A. Tropp, "CoSaMP: Iterative signal recovery from incomplete and inaccurate samples," Applied and Computational Harmonic Analysis, vol. 26, no. 3, pp. 301-321, 2009.

[44] J. A. Tropp and A. C. Gilbert, "Signal recovery from random measurements via orthogonal matching pursuit," IEEE Transactions on Information Theory, vol. 53, no. 12, pp. 4655-4666, 2007.

[45] S.-J. Kim, K. Koh, M. Lustig, S. Boyd, and D. Gorinevsky, "An interiorpoint method for large-scale $\ell_{1}$-regularized least squares," IEEE Journal of Selected Topics in Signal Processing, vol. 1, no. 4, pp. 606-617, 2007.

[46] R. Tibshirani, "Regression shrinkage and selection via the lasso," Journal of the Royal Statistical Society: Series B (Methodological), vol. 58, no. 1 , pp. 267-288, 1996.

[47] M. A. Figueiredo, R. D. Nowak, and S. J. Wright, "Gradient projection for sparse reconstruction: Application to compressed sensing and other inverse problems," IEEE Journal of Selected Topics in Signal Processing, vol. 1 , no. 4, pp. 586-597, 2007.

[48] R. Chartrand, "Exact reconstruction of sparse signals via nonconvex minimization," IEEE Signal Processing Letters, vol. 14, no. 10, pp. 707710, 2007.

[49] S. Ji, Y. Xue, and L. Carin, "Bayesian compressive sensing," IEEE Transactions on Signal Processing, vol. 56, no. 6, pp. 2346-2356, 2008.

[50] B. Sun, Y. Guo, N. Li, and D. Fang, "Multiple target counting and localization using variational bayesian em algorithm in wireless sensor networks," IEEE Transactions on Communications, vol. 65, no. 7, pp. 2985-2998, 2017.

[51] K. B. Petersen, M. S. Pedersen et al., "The matrix cookbook," Technical University of Denmark, vol. 7, no. 15, p. 510, 2008 